\title{
SPURIOUS-FREE APPROXIMATIONS OF ELECTROMAGNETIC EIGENPROBLEMS BY MEANS OF NEDELEC-TYPE ELEMENTS
}

\author{
${\text { Salvatore } \text { CaOrsi }^{1} \text {, Paolo Fernandes }}^{2}$ and Mirco Raffetto ${ }^{2}$
}

\begin{abstract}
By using an inductive procedure we prove that the Galerkin finite element approximations of electromagnetic eigenproblems modelling cavity resonators by elements of any fixed order of either Nedelec's edge element family on tetrahedral meshes are convergent and free of spurious solutions. This result is not new but is proved under weaker hypotheses, which are fulfilled in most of engineering applications. The method of the proof is new, instead, and shows how families of spurious-free elements can be systematically constructed. The tools here developed are used to define a new family of spuriousfree edge elements which, in some sense, are complementary to those defined in 1986 by Nedelec.
\end{abstract}

Mathematics Subject Classification. 65N25, 65N30, 65N12.

Received: October 23, 2000. Revised: January 29, 2001.

\section{INTRODUCTION}

The calculation of eigenfrequencies and eigenmodes of cavity resonators is so important in technological applications that the first attempts to numerically compute them date back to almost the beginning of computer age $[23,24]$. Unfortunately, after some success with scalar 2D models, more complicated ones, (no matter whether finite difference or finite element based) were often affected by extraneous solutions. In particular, some of the first finite element models of resonant cavities were totally unreliable as a consequence of the presence of the so-called spurious modes mixed with physical ones and spread over the whole numerically calculated spectrum $[15,18,22]$. Some years later, Nedelec introduced a family of elements [29] well suited to the study of $3 \mathrm{D}$ vector problems involving electromagnetic fields. These new elements (mostly the lowest order one on tetrahedra) became quite popular, usually under the name of edge elements, among researchers developing numerical simulators for the calculations of electromagnetic fields $[2,7,8]$. Very soon they realized that edge elements had solved the annoying problem of spurious modes, in all cases in which they had been used, and their popularity increased even further [9].

Even though there were clear indications that, from a practical point of view, the problem was solved, people tried to explain the correct behaviour of edge elements for essentially two reasons. The first one was to find the characteristics of edge elements which make them spurious-free so as to be able to define even new families of elements with the same good behaviour and perhaps with a better computational efficiency. The second reason

\footnotetext{
Keywords and phrases. Electromagnetic eigenproblems, new families of edge elements, Galerkin finite element approximations, convergence, spurious modes, discontinuous material properties, symmetry exploitation, mixed boundary conditions, discrete compactness.

1 Department of Electronics, University of Pavia, Via Ferrata 1, 27100 Pavia, Italy. e-mail: caorsi@ele.unipv.it

2 Istituto per la Matematica Applicata del Consiglio Nazionale delle Ricerche, Via De Marini 6, 16149 Genoa, Italy.

e-mail: fernandes@ima.ge.cnr.it, mirco@ima.ge.cnr.it
}

(c) EDP Sciences, SMAI 2001 
was to determine under which restrictions to the physical configuration, if any, the correct behaviour of edge elements takes place, so as to avoid using them when convergence is not guaranteed.

Unfortunately the first explanations of the good behaviour of edge elements $[9,17,33]$ were incomplete [10-13]. Quite recently, however, a complete theory explaining which characteristics a finite element space must have in order to guarantee convergence when used in a Galerkin approximation of an electromagnetic eigenproblem has been developed by the present authors $[13,14]$. In the meantime, other kinds of edge elements have been introduced $[17,21,30,32]$ and some properties of the edge elements defined in [29] have been proved. These properties allows to prove the convergence of the Galerkin finite element approximation based on them but the proofs usually given make use of hypotheses which are not met in problems involving discontinuous material properties or mixed boundary conditions or topologically nontrivial domains $[3-6,25,26,28]$. Thanks to the results proved in [13] the restriction on the continuity of material properties may be removed, as shown in [13] for the lowest order tetrahedral edge element, but engineers would not be fully satisfied if the other restrictions were not removed, either. As a matter of fact, they are used to exploiting symmetries to reduce computational costs $[27,35]$ but, unfortunately, this requires the introduction of mixed boundary conditions. Moreover, the region inside a practical cavity resonator may be topologically nontrivial (as, for example, in Disk-and-washer structures for particle acceleration [31], whose internal region is multiply connected and has a nonconnected boundary).

The aim of the present paper is threefold. On the one hand we give a result that ensures the convergence of Galerkin edge element approximations under assumptions that allow discontinuous material properties, topologically nontrivial domain and symmetry exploitation and are then satisfactory for most of the engineering applications. This results holds true for all (i.e., both families $[29,30]$ and any fixed order) Nedelec's elements on tetrahedral meshes and the kind of convergence we prove (spurious-free approximation) is the one expressly introduced in [13] (Def. 6.1) as the most appropriate for electromagnetic eigenproblems. On the other hand, in order to prove the aforementioned result we introduce a new approach, interesting by itself, in which the relevant properties of the elements (in particular, the discrete compactness property [25]) are proved by induction on the element order. The interesting feature of this inductive approach is that it naturally indicates how families of elements can be systematically generated in such a way that they will be spurious-free [13] by construction.

Last but not least, we exploit this fact to define a new family of spurious-free tetrahedral edge elements which, in some sense, are complementary to those defined in [30]. In 1980 Nedelec introduced [29] its first family of curl-conforming elements which provide the same order of approximation for the field and for its curl and in 1986 he defined [30] a new family which approximate better the field than its curl. The elements we introduce in this paper provide a better approximation of the curl of a vector field than of the field itself. It is important to note that it is widely recognized that the elements of the first Nedelec's family provide better results, in general. However, as pointed out by Webb [34], it is extremely important to be aware that the best element type is problem dependent. As a matter of fact, in some cases the curl may become relatively unimportant and the elements of the second Nedelec's family may outperform those of the first one [34]. In some other cases, on the contrary, the field may be less important than its curl and a global change of formulation of the problem from the electric field to the magnetic field or vice-versa may be impracticable. In these cases the new elements are expected to outperform the elements of both Nedelec's families. An extensive analysis of the performances of these elements has not been carried out so far and will be the subject of future work.

The plan of the paper is the following. In Section 2 we define the problem of interest (Sect. 2.1) and introduce two associated eigenproblems (Sect. 2.2), which will allow the treatment of discontinuous material properties and of all the boundary conditions of practical interest. The Galerkin finite element approximation is introduced in Section 3 and the characteristic conditions for the spurious-free approximation [13] are stated in Section 3.1. Two important subspaces of Nedelec's edge element spaces are introduced in Section 3.2 and the spurious-free character of the approximations by Nedelec's edge elements on tetrahedral meshes is proved in Section 3.3. Guidelines for systematically constructing families of spurious-free elements are given and new elements are introduced in Section 3.4. For a better readability of the paper many technical results, needed to work out the proofs of our main results, will be reported in the appendices. 


\section{Problem Definition}

In the applications leading to electromagnetic eigenproblems one may have to consider cavity resonators of different shapes, filled with different materials, but, as a common characteristic of all these physical situations, boundary conditions are due to conducting walls. The mathematical models of these physical devices, however, may require the use of different boundary conditions. This may happen for example because in some cases one may want to consider as primary unknown of the problem the electric field and in others the magnetic one. These two choices lead to boundary conditions of different kinds but for each specific choice one has always to deal with boundary conditions of the same type on the whole boundary. However, sometimes it is convenient to exploit the symmetries of the cavity by modelling only a part of it (referred to as a "symmetry cell") so as to reduce the computational cost $[27,35]$. In this case, the mathematical models may involve mixed boundary conditions, since a condition is enforced on the whole boundary except for some symmetry planes, where a different one is enforced. More general mixed boundary conditions cannot occur because, for either choice of the primary unknown, only one of the two considered boundary conditions is physically realizable.

\subsection{Mathematical formulation of the electromagnetic eigenproblem}

To formulate the problem in such a way to cover all the cases considered above, let us consider very general hypotheses on the cavity shape, its boundary, and the materials filling the resonator. Let $\Omega$ be an open, bounded, and connected Lipschitz polyhedron (representing the cavity resonator or a symmetry cell of it), with outward normal unit vector $\underline{n}$. Let $\Gamma=\partial \Omega$ be its boundary and assume that it splits into two disjoint open subsets $\Gamma_{\tau}$ and $\Gamma_{\nu}$, such that $\bar{\Gamma}_{\tau}$ and $\bar{\Gamma}_{\nu}$ are compact submanifolds of $\Gamma$ satisfying $\bar{\Gamma}_{\tau} \cup \bar{\Gamma}_{\nu}=\Gamma$. We ask that $\bar{\Gamma}_{\tau} \cap \bar{\Gamma}_{\nu}=\partial \Gamma_{\tau}=\partial \Gamma_{\nu}$ (if not empty) is the union of continuous piecewise straight lines. The limit cases $\Gamma_{\tau}=\emptyset$ and $\Gamma_{\nu}=\Gamma$ or $\Gamma_{\nu}=\emptyset$ and $\Gamma_{\tau}=\Gamma$ are allowed. If we are not in either limit case, then we assume that a larger domain $\tilde{\Omega} \supset \Omega$ can be generated from $\Omega$, without any overlapping, by a finite number of reflections with respect to planes containing plane parts (not necessarily connected) of either $\Gamma_{\tau}$ or $\Gamma_{\nu}$ (but not both) in such a way that $\partial \tilde{\Omega}$ originates either from $\Gamma_{\tau}$ only or from $\Gamma_{\nu}$ only. In the former case we set $\tilde{\Gamma}_{\tau}=\partial \tilde{\Omega}$, in the latter one $\tilde{\Gamma}_{\nu}=\partial \tilde{\Omega}$.

Let us assume that a set of cuts $\Sigma_{j}, j=1, \ldots, N$, satisfying the following assumptions can be introduced. Each cut $\Sigma_{j}$ is the interior of a compact and connected two-dimensional Lipschitz manifold $\bar{\Sigma}_{j}$ such that $\Sigma_{j} \subset \Omega$ and $\partial \Sigma_{j} \subset \Gamma$. Moreover, the manifolds $\bar{\Sigma}_{j}$ are mutually disjoint and for any $j$ there exists a connected open set $\Omega_{j} \supset \bar{\Sigma}_{j}$ such that $\Omega \cap \Omega_{j} \backslash \Sigma_{j}$ has exactly two connected components and each of them is a Lipschitz domain whose boundary contains $\bar{\Sigma}_{j}$. This implies in particular that the cuts are globally two-sided and the set $\dot{\Omega}=\Omega \backslash \Sigma$, with $\Sigma=\bigcup_{j=1}^{N} \Sigma_{j}$, behaves locally as a Lipschitz domain, provided that its boundary is thought as made by $\Gamma \backslash \partial \Sigma$ plus two distinct copies of $\bar{\Sigma}$. As a last hypothesis about the cuts, all of them are required in order that in $\dot{\Omega}$ every curl-free vector field has a global scalar potential. Finally, we assume that both $\Gamma_{\tau} \backslash \partial \Sigma$ and $\Gamma_{\nu} \backslash \partial \Sigma$ have a finite number of connected components and that the closure of each of them is a Lipschitz two-dimensional submanifold of $\Gamma$.

In order to model different inhomogeneous anisotropic materials filling the resonant cavity, we need to introduce two second order real symmetric tensor fields $\epsilon$ and $\mu$, defined in $\Omega$, with essentially bounded coefficients satisfying the ellipticity conditions

$$
\sum_{i, j=1}^{3} \epsilon_{i j} \xi_{i} \xi_{j} \geq \epsilon_{*}|\xi|^{2} \quad \sum_{i, j=1}^{3} \mu_{i j} \xi_{i} \xi_{j} \geq \mu_{*}|\xi|^{2} \quad \text { a. e. in } \Omega, \forall \xi \in \mathbb{R}^{3}
$$

for two positive constants $\epsilon_{*}$ and $\mu_{*}$. We also assume that $\Omega$ can be split into disjoint Lipschitz open subdomains $\Omega_{k}, k=1, \ldots, r$, such that $\bigcup_{k=1}^{r} \bar{\Omega}_{k}=\bar{\Omega}$ and all the coefficients of $\epsilon$ and $\mu$ (which may be globally discontinuous) are Lipschitz continuous in each of them. Notice that these assumptions will allow us to use results in [19] and [13]. 
With these notations, the electromagnetic eigenproblem modelling cavity resonators has the following standard variational formulation, which, as well known, has extraneous solutions with respect to the Maxwell system, but does not involve a divergence-free constraint on the variational space [6]:

Problem 1. Find $(\omega, \underline{u} \neq 0) \in \mathbb{R} \times V$ :

$$
\left(\mu^{-1} \operatorname{curl} \underline{u}, \operatorname{curl} \underline{v}\right)_{0, \Omega}=\omega^{2}(\epsilon \underline{u}, \underline{v})_{0, \Omega} \forall \underline{v} \in V
$$

where

$$
V=H_{0, \Gamma_{\tau}}(\operatorname{curl} ; \Omega)=\left\{\underline{v} \in L^{2}(\Omega)^{3}\left|\operatorname{curl} \underline{v} \in L^{2}(\Omega)^{3}, \underline{v} \times \underline{n}\right|_{\Gamma_{\tau}}=0\right\}
$$

and $(,)_{0, \Omega}$ indicates the standard scalar product in $H=L^{2}(\Omega)^{3}$.

Problem 1 covers all cases we are interested in. If $\underline{u}$ is the electric field, then $\Gamma_{\tau}$ models both ideally conducting walls and skew-symmetry planes of $\underline{u}$ (see Sect. 2.2), while $\Gamma_{\nu}$ models symmetry planes of $\underline{u}$ (see Sect. 2.2). If $\underline{u}$ is the magnetic field, instead, then conducting walls are modelled by $\Gamma_{\nu}$, while modelling of symmetries (with respect to $\underline{u}$ ) is unchanged.

We will also need the following spaces (see [19]):

$$
\begin{aligned}
& V_{0}=H_{0, \Gamma_{\tau}}\left(\operatorname{curl}^{0} ; \Omega\right)=\{\underline{v} \in V \mid \operatorname{curl} \underline{v}=0\} \\
& H_{0, \Gamma_{\nu}}\left(\operatorname{div}^{0}, \Omega, \epsilon\right)=\left\{\underline{v} \in L^{2}(\Omega)^{3}|\operatorname{div} \epsilon \underline{v}=0, \epsilon \underline{v} \cdot \underline{n}|_{\Gamma_{\nu}}=0\right\} \\
& H_{1}=\epsilon^{-1} \operatorname{curl}\left(H_{0, \Gamma_{\nu}}(\operatorname{curl}, \Omega)\right) \subset H_{0, \Gamma_{\nu}}\left(\operatorname{div}^{0}, \Omega, \epsilon\right) \\
& V_{1}=V \cap H_{1} \\
& \mathbb{H}=\mathbb{H}\left(\Omega, \Gamma_{\tau}, \epsilon\right)=H_{0, \Gamma_{\tau}}\left(\operatorname{curl}^{0}, \Omega\right) \cap H_{0, \Gamma_{\nu}}\left(\operatorname{div}^{0}, \Omega, \epsilon\right) \\
& H_{0, \Gamma_{\tau}}^{1}(\Omega)=\left\{\phi \in L^{2}(\Omega)\left|\operatorname{grad} \phi \in L^{2}(\Omega)^{3}, \phi\right|_{\Gamma_{\tau}}=0\right\} .
\end{aligned}
$$

We will indicate by \|\|$_{0, \Omega}$ the norm in $H$ corresponding to $(,)_{0, \Omega}$, by $(,)_{\text {curl }, \Omega}$ and \|\|$_{\text {curl }, \Omega}$ the standard inner product and norm in $H(\operatorname{curl}, \Omega)$, respectively, and by \|\|$_{s, \Omega}, 0<s \leq 1$, the natural norm in $H^{s}(\Omega)$ or $H^{s}(\Omega)^{3}$. For $s=1$ we will also use the natural seminorm ||$_{1, \Omega}[16]$. Finally, we define the following inner products and norms in $H$ and $V$ :

$$
\begin{aligned}
(\underline{u}, \underline{v})_{H, \Omega} & =(\epsilon \underline{u}, \underline{v})_{0, \Omega}, \forall \underline{u} \in H, \forall \underline{v} \in H \\
\|\underline{v}\|_{H, \Omega} & =(\underline{v}, \underline{v})_{H, \Omega}^{1 / 2} \forall \underline{v} \in H \\
(\underline{u}, \underline{v})_{V, \Omega} & =(\epsilon \underline{u}, \underline{v})_{0, \Omega}+\left(\mu^{-1} \operatorname{curl} \underline{u}, \operatorname{curl} \underline{v}\right)_{0, \Omega}, \forall \underline{u} \in V, \forall \underline{v} \in V \\
\|\underline{v}\|_{V, \Omega} & =(\underline{v}, \underline{v})_{V, \Omega}^{1 / 2}, \forall \underline{v} \in V .
\end{aligned}
$$

Owing to the assumptions about $\epsilon$ and $\mu,\|\|_{H, \Omega}$ is equivalent to \|\|$_{0, \Omega}$ while \|\|$_{V, \Omega}$ is equivalent to \|\|$_{\text {curl, } \Omega}$. Whenever nothing different is explicitly stated, it is understood that $H$ and $V$ are endowed with the inner products $(\underline{u}, \underline{v})_{H, \Omega}$ and $(\underline{u}, \underline{v})_{V, \Omega}$ (and the corresponding norms), respectively, which make them Hilbert spaces. The most widely used subspaces of $H$ and $V$, respectively, will take the structure induced by the space $(H$ or $V$, respectively) containing them. 
The following decompositions, orthogonal in both $H$ and $V$, hold true (see Prop. 2.2 of [13], and [19])

$$
\begin{aligned}
& V=V_{0} \oplus V_{1} \\
& V_{0}=\operatorname{grad}\left(H_{0, \Gamma_{\tau}}^{1}(\Omega)\right) \oplus \mathbb{H} \\
& H_{0, \Gamma_{\nu}}\left(\operatorname{div}^{0}, \Omega, \epsilon\right)=\mathbb{H} \oplus H_{1}
\end{aligned}
$$

and $\mathbb{H}$ is reduced to $\{0\}$ when both $\Omega$ and $\Gamma_{\tau}$ are topologically trivial.

\subsection{Two related eigenproblems}

We will consider also two other eigenvalue problems related to Problem 1 . The first one is formulated exactly as Problem 1 except for $\epsilon=1=\mu$ ( 1 is the 3-by-3 identity matrix).

Problem 2. The same as Problem 1, but with $\epsilon=1=\mu$.

The sets $\Omega, \Gamma, \Gamma_{\tau}$, and $\Gamma_{\nu}$ and the spaces $V$ and $V_{0}$ for Problem 2 are the same as those of Problem 1 and, for this reason, we will use the same notations. However, the spaces defined in (5), (6) and (7) are now different. These spaces will be indicated by $H_{1, p b 2}, V_{1, p b 2}$ and $\mathbb{H}_{p b 2}$.

The second related eigenproblem we introduce is posed in the domain $\tilde{\Omega}$ defined in Section 2.1. For this problem we will exactly use the same notations as for Problem 1 except for a $\sim$ on the top of each symbol.

Problem 3. The same as Problem 2, but posed in the domain $\tilde{\Omega}$, hence with either $\tilde{\Gamma}_{\nu}=\emptyset$ and $\tilde{\Gamma}=\tilde{\Gamma}_{\tau}$ or $\tilde{\Gamma}_{\tau}=\emptyset$ and $\tilde{\Gamma}=\tilde{\Gamma}_{\nu}$.

If $\Gamma_{\nu}=\emptyset$ or $\Gamma_{\tau}=\emptyset$ in Problem 1 , then we could simply choose $\tilde{\Omega}=\Omega$ as well, even if $\Omega$ does not represent the whole cavity. Otherwise, since in Problem 1 we allow mixed boundary conditions only to exploit symmetries, $\tilde{\Omega}$ represents the (unloaded) cavity resonator and $\Omega$ is a symmetry cell of $\tilde{\Omega}$, namely a subdomain in which the problem is posed on the basis of symmetry considerations. In practice, in most cases the whole cavity is obtained from $\Omega$ by a finite number of reflections with respect to planes. Even though also other kinds of symmetries could be considered and dealt with in a similar way, we will content ourselves with this case, which covers most of the applications.

Now, let us recall some basic facts about symmetry. A plane $S$ is a symmetry plane for a subset of $\mathbb{R}^{3}$ if this set is left unchanged by a reflection with respect to $S$. A vector field $\underline{v}: \Omega \rightarrow \mathbb{R}^{3}$ is said symmetrical (respectively skew-symmetrical) with respect to $S$, symmetry plane of $\Omega$, if it is left unchanged (respectively, just reversed) by a reflection with respect to $S$. Let us introduce $x, y, z$ orthogonal axes in such a way that $S$ is the plane $z=0$. Then, it is not difficult to check the following properties of (skew-) symmetrical vector fields. A vector field $\underline{v}(x, y, z)$ is symmetrical with respect to $S$ if and only if $v_{x}(x, y,-z)=v_{x}(x, y, z)$, $v_{y}(x, y,-z)=v_{y}(x, y, z), v_{z}(x, y,-z)=-v_{z}(x, y, z), \forall(x, y, z) \in \Omega$. It is skew-symmetrical if and only if $v_{x}(x, y,-z)=-v_{x}(x, y, z), v_{y}(x, y,-z)=-v_{y}(x, y, z), v_{z}(x, y,-z)=v_{z}(x, y, z), \forall(x, y, z) \in \Omega$. If $\underline{v}$ is symmetrical (respectively, skew-symmetrical), then curl $\underline{v}$ is skew-symmetrical (respectively, symmetrical). Let $\underline{v}$ be symmetrical. If $\underline{u}$ is symmetrical (respectively, skew-symmetrical) $\underline{u} \times \underline{v}$ is skew-symmetrical (respectively, symmetrical). By exploiting the above properties we can prove the following lemma.

Lemma 1. Let $S$ be a symmetry plane for $\Omega$ and $\Gamma_{\tau}$ (hence, also for $\Gamma_{\nu}$ ) and let $\Gamma_{S}=S \cap \Omega$ subdivide $\Omega$ in two specular subdomains $\Omega_{1}$ and $\Omega_{2}$. Then, any eigenvector $\underline{u}_{1}$ of Problem 2 posed in $\Omega_{1}$ with $\underline{u}_{1} \times\left.\underline{n}_{1}\right|_{\Gamma_{S}}=0$ (respectively, curl $\underline{u}_{1} \times\left.\underline{n}_{1}\right|_{\Gamma_{S}}=0$ ) is the restriction of a skew-symmetrical (respectively, symmetrical) eigenvector $\underline{u}$ of Problem 2 posed in $\Omega$. Moreover, $\|\underline{u}\|_{\text {curl }, \Omega}=\sqrt{2}\left\|\underline{u}_{1}\right\|_{\text {curl }, \Omega_{1}}$.

Proof. It is standard matter to show the following two equivalences. Problem 2 posed in $\Omega$ is equivalent to the transmission problem curl curl $\underline{u}=\omega^{2} \underline{u}$ a.e. in $\Omega_{1}$ and $\Omega_{2} ; \underline{u} \times\left.\underline{n}\right|_{\Gamma_{\tau}}=0 ; \operatorname{curl} \underline{u} \times\left.\underline{n}\right|_{\Gamma_{\nu}}=0 ; \underline{u}_{1} \times\left.\underline{n}_{1}\right|_{\Gamma_{S}}+\underline{u}_{2} \times$ $\left.\underline{n}_{2}\right|_{\Gamma_{S}}=0 ; \operatorname{curl} \underline{u}_{1} \times\left.\underline{n}_{1}\right|_{\Gamma_{S}}+\operatorname{curl} \underline{u}_{2} \times\left.\underline{n}_{2}\right|_{\Gamma_{S}}=0$. Problem 2 posed in $\Omega_{1}$ with $\underline{u}_{1} \times\left.\underline{n}_{1}\right|_{\Gamma_{S}}=0$ is equivalent to curl curl $\underline{u}_{1}=\omega^{2} \underline{u}_{1}$ a.e. in $\Omega_{1} ; \underline{u}_{1} \times\left.\underline{n}\right|_{\Gamma_{\tau} \cap \partial \Omega_{1}}=0$; curl $\underline{u}_{1} \times\left.\underline{n}\right|_{\Gamma_{\nu} \cap \partial \Omega_{1}}=0 ; \underline{u}_{1} \times\left.\underline{n}_{1}\right|_{\Gamma_{S}}=0$. Suppose the solution $\underline{u}_{1}$ of the problem in $\Omega_{1}$ is extended to a vector field $\underline{u}$ defined in $\Omega$, skew-symmetrical with respect to $S$ and 
such that $\left.\underline{u}\right|_{\Omega_{1}}=\underline{u}_{1}$. By the previously stated properties of (skew-) symmetrical vector fields and taking into account that $\underline{n}$ is symmetrical, it is straightforward to verify that the extended vector field $\underline{u}$ satisfies the above transmission problem. The case with curl $\underline{u}_{1} \times\left.\underline{n}_{1}\right|_{\Gamma_{S}}=0$ in place of $\underline{u}_{1} \times\left.\underline{n}_{1}\right|_{\Gamma_{S}}=0$ is worked out in the same way except that $\underline{u}_{1}$ is extended to a symmetrical (rather than skew-symmetrical) $\underline{u}$. In both cases, exploiting symmetries, we obtain $\|\underline{u}\|_{\text {curl }, \Omega}^{2}=\|\underline{u}\|_{0, \Omega}^{2}+\|\operatorname{curl} \underline{u}\|_{0, \Omega}^{2}=2\left\|\underline{u}_{1}\right\|_{0, \Omega_{1}}^{2}+2\left\|\operatorname{curl} \underline{u}_{1}\right\|_{0, \Omega_{1}}^{2}=2\left\|\underline{u}_{1}\right\|_{\text {curl }, \Omega_{1}}^{2}$

We are now in a position to prove the following lemma.

Lemma 2. Any eigenvector $\underline{u}$ of Problem 2 is the restriction to $\Omega$ of some eigenvector $\underline{\tilde{u}}$ of Problem 3. Moreover $\underline{\tilde{u}}$ can be obtained from $\underline{u}$ by some finite number $m$ of extensions to specular domains by symmetry or skewsymmetry and $\|\underline{\tilde{u}}\|_{\mathrm{curl}, \tilde{\Omega}}=2^{m / 2}\|\underline{u}\|_{\mathrm{curl}, \Omega}$.

Proof. Since $\tilde{\Omega}$ is obtained from $\Omega$ by a finite number of reflections with respect to a plane, this lemma is an easy consequence of repeated applications of Lemma 1.

The following lemmas establish very useful regularity properties of the spaces $V_{1, p b 2}, \tilde{V}_{1}, \mathbb{H}_{p b 2}$ and $\tilde{\mathbb{H}}$.

Lemma 3. There exists a real number $s>1 / 2$ such that $\tilde{V}_{1} \hookrightarrow H^{s}(\tilde{\Omega})^{3}$ and $\tilde{\mathbb{H}} \hookrightarrow H^{s}(\tilde{\Omega})^{3}$.

Proof. We know that $\epsilon=1=\mu$. Moreover, either $\tilde{\Gamma}_{\nu}=\emptyset$ and $\tilde{\Gamma}=\tilde{\Gamma}_{\tau}$ or $\tilde{\Gamma}_{\tau}=\emptyset$ and $\tilde{\Gamma}=\tilde{\Gamma}_{\nu}$. Then we can apply Proposition 3.7 of [1] from which both continuous embeddings follow.

Lemma 4. If $\tilde{V}_{1} \hookrightarrow H^{s}(\tilde{\Omega})^{3}$ for some $s>1 / 2$ then $V_{1, p b 2} \hookrightarrow H^{s}(\Omega)^{3}$ for the same value of $s$.

Proof. Given $\underline{v} \in V_{1, p b 2}$ by Proposition 3.4 of [13] we have $\underline{v}=\sum_{i=1}^{\infty}\left(\underline{v}, \underline{v}_{i}\right)_{\operatorname{curl}, \Omega} \underline{v}_{i}$, where $\underline{v}_{i}, i=1, \cdots, \infty$, is an eigenvector of Problem 2. By Lemma 2, for any $\underline{v}_{i}$ there exists an eigenvector $\underline{\underline{v}}_{i}$ of Problem 3 such that $\left.\underline{\underline{v}}_{i}\right|_{\Omega}=\underline{v}_{i}$. Again by Proposition 3.4 of [13], the vector field $\underline{\tilde{v}}=\sum_{i=1}^{\infty}\left(\underline{v}, \underline{v}_{i}\right)_{\operatorname{curl}, \Omega} \underline{\underline{v}}_{i}$ belongs to $\tilde{V}_{1}$ and, thus, to $H^{s}(\tilde{\Omega})^{3}$ for some $s>1 / 2$. Then $\underline{v}=\left.\underline{\tilde{v}}\right|_{\Omega} \in H^{s}(\Omega)^{3}$ for the same $s>1 / 2$. Thus, $V_{1, p b 2} \subset H^{s}(\Omega)^{3}$ for the same $s>1 / 2$. Moreover, $\exists C>0$ such that $\|\underline{\tilde{v}}\|_{s, \tilde{\Omega}} \leq C\|\underline{\tilde{v}}\|_{\operatorname{curl}, \tilde{\Omega}} \forall \underline{\tilde{v}} \in \tilde{V}_{1}$. Then $\|\underline{v}\|_{s, \Omega}=\left\|\left.\underline{\tilde{v}}\right|_{\Omega}\right\|_{s, \Omega} \leq\|\underline{\tilde{v}}\|_{s, \tilde{\Omega}} \leq C\|\underline{\tilde{v}}\|_{\operatorname{curl}, \tilde{\Omega}}$. But $\underline{\underline{v}}$ is obtained from $\underline{v}$ through the same finite sequence of symmetrical or skew-symmetrical extensions giving each $\underline{\tilde{v}}_{i}$ from the corresponding $\underline{v}_{i}$ (see Lem. 2). Then, $\|\underline{\tilde{\tilde{v}}}\|_{\mathrm{curl}, \tilde{\Omega}}=2^{m / 2}\|\underline{v}\|_{\mathrm{curl}, \Omega}$, where $m$ is the number of extensions, and, consequently $\|\underline{v}\|_{s, \Omega} \leq C 2^{m / 2}\|\underline{v}\|_{\text {curl }, \Omega}$. Hence, the embedding is continuous.

Lemma 5. If $\tilde{\mathbb{H}} \hookrightarrow H^{s}(\tilde{\Omega})^{3}$ for some $s>1 / 2$ then $\mathbb{H}_{p b 2} \hookrightarrow H^{s}(\Omega)^{3}$ for the same value of $s$.

Proof. Completely analogous to the proof of Lemma 4 except that, as $\underline{v} \in \mathbb{H}_{p b 2}$ is already an eigenvector of Problem 2, no series expansion is needed.

\section{GALERKIn Finite ELEMENT APPROXIMATIONS}

A curl-conforming Galerkin finite element approximation of Problem 1 is obtained by introducing a family of triangulations $\left\{\mathcal{T}_{h}\right\}_{h \in I}$ of $\bar{\Omega}$ and a specific finite element on the triangulation $\mathcal{T}_{h}$, so defining a family $\left\{V_{h}\right\}_{h \in I}$ of finite dimensional subspaces of $V$. We will assume, as usual, that $h$ denotes the maximum diameter of all elements of the triangulation $\mathcal{T}_{h}$, that $I$ is a denumerable and bounded set of strictly positive numbers having zero as the only limit point, that the family of triangulations is regular [16], that, for every $h, \mathcal{T}_{h}$ exactly covers $\bar{\Omega}$ (i.e., $\bar{\Omega}=\cup_{K \in \mathcal{T}_{h}} K$ ), that, for every $h, \bar{\Gamma}_{\nu}$ and $\bar{\Gamma}_{\tau}$ are the union of faces of elements of $\mathcal{T}_{h}$. We will also assume [1] that for each $h$, there exists a set of $J$ cuts $\Sigma_{j, h}, 1 \leq j \leq J$, satisfying the assumptions of Section 2.1 and such that each $\bar{\Sigma}_{j, h}$ is the union of faces of elements of $\mathcal{T}_{h}$. Thus, we can define $\Sigma_{h}=\bigcup_{j=1}^{N} \Sigma_{j, h}$ and $\dot{\Omega}_{h}=\Omega \backslash \Sigma_{h}$. Moreover, it is understood that (the integrals defining) the scalar products involved in the Galerkin finite element approximations are exactly evaluated. 
Then, the Galerkin finite element approximations of Problem 1 read as follows:

Problem 4. Find $\left(\omega_{h}, \underline{u}_{h} \neq 0\right) \in \mathbb{R} \times V_{h}$ :

$$
\left(\mu^{-1} \operatorname{curl} \underline{u}_{h}, \operatorname{curl} \underline{v}_{h}\right)_{0, \Omega}=\omega_{h}^{2}\left(\epsilon \underline{u}_{h}, \underline{v}_{h}\right)_{0, \Omega} \forall \underline{v}_{h} \in V_{h} .
$$

Owing to the assumptions concerning exact evaluation of the scalar products, the most general material properties easily dealt with, in practice, are globally discontinuous tensor fields, piecewise polynomial on Lipschitz polyhedral subdomains. In order to make actually easy the treatment of this case, for every $h$, the closure of each of these subdomains must be exactly covered by the union of elements of $\mathcal{T}_{h}$.

We will also consider the discrete eigenproblem defined exactly as Problem 4 except for $\epsilon=1=\mu$.

Problem 5. The same formulation as Problem 4 but with $\epsilon=1=\mu$.

Note that Problem 5 can be regarded as a Galerkin finite element approximation of Problem 2. Also in this case we will use the subscript $p b 5$ only to denote the discrete subspaces which are different from the corresponding ones of Problem 4.

\subsection{Characteristic conditions for the spurious free approximation}

Given $\left\{X_{h}\right\}, X_{h} \subset V \forall h \in I$, let $X_{0 h}=X_{h} \cap V_{0}$ and $X_{1 h}$ be the orthogonal complement of $X_{0 h}$ in $X_{h}$ with respect to the $(,)_{V, \Omega}$ scalar product. We define the following conditions on the sequence $\left\{X_{h}\right\}[13]$

(CAS) "Completeness of the approximating subspace"

$$
\lim _{h \rightarrow 0} \inf _{h} \in X_{h}\left\|\underline{v}-\underline{x}_{h}\right\|_{V, \Omega}=0 \forall \underline{v} \in V
$$

(CDK) "Completeness of the discrete kernel"

$$
\lim _{h \rightarrow 0} \inf _{h} \in X_{0 h}\left\|\underline{v}-\underline{x}_{h}\right\|_{V, \Omega}=0 \forall \underline{v} \in V_{0}
$$

(DCP) "Discrete compactness property"

any sequence $\left\{\underline{x}_{h}\right\}_{h \in I}$, such that $\underline{x}_{h} \in X_{1 h},\left\|\underline{x}_{h}\right\|_{V, \Omega} \leq C \forall h \in I$, contains a sub-sequence (still denoted by $\left.\left\{\underline{x}_{h}\right\}\right)$ such that $\exists \underline{v} \in H$ :

$$
\lim _{h \rightarrow 0}\left\|\underline{x}_{h}-\underline{v}\right\|_{H, \Omega}=0 .
$$

These conditions play a crucial role in the analysis of the convergence of the finite element approximations of Problem 1. As a matter of fact, it is proved in [13] that it is necessary and sufficient that $\left\{V_{h}\right\}$ satisfies the above three characteristic conditions in order that Problem 4 can be regarded as a "spurious-free approximation" [13] of the following restriction of Problem 1, which provides all the significant (i.e., non static, namely having $\omega \neq 0)$ solutions of the Maxwell system without introducing any extraneous one:

Problem 6. Find $(\omega, \underline{u} \neq 0) \in \mathbb{R} \times V_{1}$ :

$$
\left(\mu^{-1} \operatorname{curl} \underline{u}, \operatorname{curl} \underline{v}\right)_{0, \Omega}=\omega^{2}(\epsilon \underline{u}, \underline{v})_{0, \Omega} \forall \underline{v} \in V_{1} .
$$

In the present paper we will apply this result to all the elements of the Nedelec's edge element families by proving that they generate sequences of spaces $\left\{V_{h}\right\}$ that satisfy the above three characteristic conditions. We will actually achieve this target in an indirect way, by exploiting again some results proved in [13]. As a matter of fact, it is proved in [13] that if the sequence of spaces $\left\{V_{h}\right\}$ satisfies (CAS), (CDK), and (DCP) for $\epsilon=1=\mu$ (involved in Problems 2 and 5), then it satisfies the same conditions for $\epsilon$ and $\mu$ involved in both Problems 1 
and 4. Therefore, we will achieve our target by proving that all elements of the Nedelec's edge element families generate sequences of spaces $\left\{V_{h}\right\}$ that satisfy (CAS), (CDK), and (DCP) for $\epsilon=1=\mu$, exactly as we were concerned just with the behaviour of Problem 5 as an approximation of Problem 2.

\subsection{Subspaces of Nedelec's tetrahedral edge elements}

Let $P_{l}$ be the space of polynomials of degree at most $l, \tilde{P}_{l}$ be the space of homogeneous polynomials of degree $l$, and $B\left(P_{l}\right)$ be a basis of $P_{l}$. Moreover, let $K$ be a tetrahedron in $\mathbb{R}^{3}, E$ be the set of its edges and $F$ be the set of its faces. The space of classic edge element functions defined by Nedelec [29] is: $R_{l}=P_{l-1}^{3} \oplus S_{l}$ where $S_{l}=\left\{\underline{s} \in \tilde{P}_{l}^{3}: \underline{s} \cdot \underline{x}=0\right\}$ and $l>0$, whereas the space of more recent edge element functions [30] is: $Q_{l}=P_{l}^{3}$, $l>0$. Note that from these definitions we immediately obtain $R_{l+1}=Q_{l} \oplus S_{l+1}, l>0$. A complete definition of the Nedelec's edge element families $[29,30]$ can be carried out by considering the following sets of moments of a function $\underline{u} \in W^{1, s}(K)^{3}, s>2[20]$, for the space $R_{l}, l>0$ :

$$
M_{E, R, l}(\underline{u})=\left\{\int_{e}(\underline{u} \cdot \underline{\tau}) q, \forall q \in B\left(P_{l-1}(e)\right), \forall e \in E\right\}
$$

where $\underline{\tau}$ denotes the unit vector of $e, e \in E$,

$$
M_{F, R, l}(\underline{u})= \begin{cases}\left\{\int_{f}(\underline{u} \times \underline{n}) \cdot \underline{q}, \forall \underline{q} \in B\left(P_{l-2}^{2}(f)\right), \forall f \in F\right\} & \text { if } l>1 \\ \emptyset & \text { if } l=1\end{cases}
$$

where $\underline{n}$ denotes the outward unit vector normal to $f, f \in F$, and

$$
M_{K, R, l}(\underline{u})= \begin{cases}\left\{\int_{K} \underline{u} \cdot \underline{q}, \forall \underline{q} \in B\left(P_{l-3}^{3}(K)\right)\right\} & \text { if } l>2 \\ \emptyset & \text { if } l=1,2\end{cases}
$$

and the following sets of moments for the space $Q_{l}[30], l>0$ :

$$
\begin{aligned}
& M_{E, Q, l}(\underline{u})=\left\{\int_{e}(\underline{u} \cdot \underline{\tau}) q, \forall q \in B\left(P_{l}(e)\right), \forall e \in E\right\} \\
& M_{F, Q, l}(\underline{u})= \begin{cases}\left\{\int_{f} \underline{u} \cdot \underline{q}, \forall \underline{q} \in B\left(D_{l-1}(f)\right), \forall f \in F\right\} & \text { if } l>1 \\
\emptyset & \text { if } l=1\end{cases} \\
& M_{K, Q, l}(\underline{u})= \begin{cases}\left\{\int_{K} \underline{u} \cdot \underline{q}, \forall \underline{q} \in B\left(D_{l-2}(K)\right)\right\} & \text { if } l>2 \\
\emptyset & \text { if } l=1,2\end{cases}
\end{aligned}
$$

where $D_{l}(K)=P_{l-1}^{3}(K) \oplus \underline{x} \tilde{P}_{l-1}(K), \underline{x}$ is the position vector $\underline{x}=(x, y, z), D_{l}(f)=P_{l-1}^{2}(f) \oplus \underline{x} \tilde{P}_{l-1}(f)$, and $B\left(D_{l}\right)$ represents a basis of $D_{l}$. It is understood that the moments on edges (respectively, faces) and the involved polynomial spaces are defined with respect to 1D (respectively, 2D) Cartesian coordinates local to 
the edge (respectively, face). In particular, all the elements of $P_{l}^{2}(f), \underline{x}$ in the definition of $D_{l}(f)$ and all the elements of $D_{l}(f)$ itself are vector fields tangent to $f$ (see [30] p. 68).

The following shorter notations will also be useful:

$$
\begin{aligned}
& M_{R, l}(\underline{u})=M_{E, R, l}(\underline{u}) \cup M_{F, R, l}(\underline{u}) \cup M_{K, R, l}(\underline{u}) \\
& M_{Q, l}(\underline{u})=M_{E, Q, l}(\underline{u}) \cup M_{F, Q, l}(\underline{u}) \cup M_{K, Q, l}(\underline{u}) .
\end{aligned}
$$

For these families of elements Nedelec $[29,30]$ proved a lot of interesting results. The following six lemmas report only those of particular importance for our analysis. Let $G_{l}=\left\{\nabla \varphi: \varphi \in \tilde{P}_{l+1}\right\}, G_{l} \subset \tilde{P}_{l}^{3}$.

Lemma 6. $G_{l} \cap S_{l}=\{0\}$ and $G_{l} \oplus S_{l}=\tilde{P}_{l}^{3}$.

Proof. See [29] (p. 322).

Corollary 1. $Q_{l}=P_{l-1}^{3} \oplus \tilde{P}_{l}^{3}=P_{l-1}^{3} \oplus S_{l} \oplus G_{l}=R_{l} \oplus G_{l}$.

Lemma 7. $\underline{u} \in S_{l}$ and curl $\underline{u}=0$ implies $\underline{u}=0$.

Proof. See [29] (p. 321).

Lemma 8. Any vector field $\underline{u} \in Q_{l}$ having all the moments in $M_{R, l}(\underline{u})$ equal to zero is irrotational.

Proof. See [29] (p. 323).

Lemma 9. Any vector field $\underline{u} \in R_{l+1}$ satisfying curl $\underline{u}=0$ actually belongs to $Q_{l}$.

Proof. It is proved in [20] (p. 263) that $\underline{u}=\operatorname{grad} p$ with $p \in P_{l+1}$ and this implies $\underline{u} \in Q_{l}$.

Lemma 10. The set of moments $M_{R, l}(\underline{u})$ uniquely identify the vector field $\underline{u} \in R_{l}$. Moreover, the tangential components of $\underline{u}$ on a given face $f$ of $K$ depend only on the moments belonging to $M_{E, R, l}(\underline{u})$ and $M_{F, R, l}(\underline{u})$ defined on that face.

Proof. See [29] (p. 326).

Lemma 11. The set of moments $M_{Q, l}(\underline{u})$ uniquely identify the vector field $\underline{u} \in Q_{l}$. Moreover, the tangential components of $\underline{u}$ on a given face $f$ of $K$ depend only on the moments belonging to $M_{E, Q, l}(\underline{u})$ and $M_{F, Q, l}(\underline{u})$ defined on that face.

Proof. See [30] (p. 68).

Moreover, from the above definitions of the sets of moments (16)-(21), we deduce:

Lemma 12. With a suitable choice of the basis, the three sets of moments of a vector field $\underline{u} \in Q_{l}, M_{E, R, l}(\underline{u})$, $M_{F, R, l}(\underline{u}), M_{K, R, l}(\underline{u})$, are subsets, respectively, of the moments $M_{E, Q, l}(\underline{u}), M_{F, Q, l}(\underline{u}), M_{K, Q, l}(\underline{u})$.

Proof. As $P_{l-1}(e) \subset P_{l}(e) \forall e \in E$, we can choose $B\left(P_{l}(e)\right) \supset B\left(P_{l-1}(e)\right)$ and then $M_{E, R, l}(\underline{u}) \subset M_{E, Q, l}(\underline{u})$. In the same way, $M_{K, R, l}(\underline{u}) \subset M_{K, Q, l}(\underline{u})$ as $P_{l-3}^{3}(K) \subset D_{l-2}(K)$. Moreover, $(\underline{u} \times \underline{n}) \cdot \underline{q}=\underline{u} \cdot(\underline{n} \times \underline{q})$ and $(\underline{n} \times \underline{q})$ belongs to $P_{l-2}^{2}(f)$ if $\underline{q}$ does. Hence, as $P_{l-2}^{2}(f) \subset D_{l-1}(f) \forall f \in F$ we also obtain $\bar{M}_{F, R, l}(\underline{u}) \subset M_{F, Q, l}(\underline{u})$.

Lemma 13. With a suitable choice of the basis, the three sets of moments of a vector field $\underline{u} \in R_{l+1}, M_{E, Q, l}(\underline{u})$, $M_{F, Q, l}(\underline{u}), M_{K, Q, l}(\underline{u})$, are subsets, respectively, of the moments $M_{E, R, l+1}(\underline{u}), M_{F, R, l+1}(\underline{u}), M_{K, R, l+1}(\underline{u})$.

Proof. $M_{E, R, l+1}(\underline{u})=M_{E, Q, l}(\underline{u})$ by simply using the same basis, as the space of polynomials involved is the same. As $D_{l-2}(K) \subset P_{l-2}^{3}(K)$, we can choose $B\left(P_{l-2}^{3}(K)\right) \supset B\left(D_{l-2}(K)\right)$ and then $M_{K, Q, l}(\underline{u}) \subset M_{K, R, l+1}(\underline{u})$. Finally, as $D_{l-1}(f) \subset P_{l-1}^{2}(f) \forall f \in F$ we also obtain $M_{F, Q, l}(\underline{u}) \subset M_{F, R, l+1}(\underline{u})$. 
In the following we will always assume that the basis of the polynomial spaces have been chosen in such a way that the conclusions of Lemmas 12 and 13 hold true.

In order to devise some crucial additional properties of the spaces of vector fields involved in the definitions of the Nedelec's edge element families, let us consider $q \in Q_{l}$ and let $M_{Q, l}(q)$ be the corresponding moments. Moreover, let $\underline{r}(\underline{q})$ be the unique vector field in $R_{l}$ such that $M_{R, l}(\underline{r}(\underline{q}))=M_{R, l}(\underline{q})$. It is then possible to define $\underline{u}_{1}(\underline{q})=\underline{q}-\underline{r}(\underline{q}) \in Q_{l}$. All the moments in $M_{R, l}\left(\underline{u}_{1}\right)$ are equal to zero. It is then convenient to define the following important spaces:

Definition 1. For any $l>0, F_{l}$ is the subspace of the elements $\underline{u}$ of $Q_{l}$ such that all the moments in $M_{R, l}(\underline{u})$ vanish.

Both the linear and everywhere defined operators $\underline{r}(\underline{q}): Q_{l} \rightarrow R_{l} \subset Q_{l}$ and $\underline{u}_{1}(\underline{q}): Q_{l} \rightarrow F_{l} \subset Q_{l}$ are surjective since $\underline{r}\left(R_{l}\right)=R_{l}$ and $\underline{u}_{1}\left(F_{l}\right)=F_{l}$. Hence, as $\underline{q}=\underline{r}(\underline{q})+\underline{u}_{1}(\underline{q})$ we obtain $Q_{l}=R_{l}+F_{l}$.

In an analogous way, we can also consider $\underline{r} \in R_{l+1}$. If $M_{R, l+1}(\underline{r})$ are the moments identifying $\underline{r}$, let $\underline{q}(\underline{r})$ be the unique vector field in $Q_{l}$ such that $M_{Q, l}(\underline{q}(\underline{r}))=M_{Q, l}(\underline{r})$. It is then possible to define $\underline{u}_{2}(\underline{r})=\underline{r}-\underline{q}(\underline{r}) \in$ $R_{l+1}$. All the moments in $M_{Q, l}\left(\underline{u}_{2}\right)$ are equal to zero. Then, we conveniently define the following important spaces:

Definition 2. For any $l>0, E_{l+1}$ is the subspace of the elements $\underline{u}$ of $R_{l+1}$ such that all the moments in $M_{Q, l}(\underline{u})$ vanish.

Both the linear and everywhere defined operators $\underline{q}(\underline{r}): R_{l+1} \rightarrow Q_{l} \subset R_{l+1}$ and $\underline{u}_{2}(\underline{r}): R_{l+1} \rightarrow E_{l+1} \subset R_{l+1}$ are surjective since $q\left(Q_{l}\right)=Q_{l}$ and $\underline{u}_{2}\left(E_{l+1}\right)=E_{l+1}$. Hence, as $\underline{r}=\underline{q}(\underline{r})+\underline{u}_{2}(\underline{r})$ we obtain $R_{l+1}=Q_{l}+E_{l+1}$.

The following four lemmas point out some important properties of the subspaces $F_{l}$ and $E_{l+1}$ just defined, and provide alternative representations of the spaces $Q_{l}$ and $R_{l}$.

Lemma 14. $Q_{l}=R_{l} \oplus F_{l}$.

Proof. We have only to prove that the sum is actually a direct sum. By contradiction, let us suppose $\exists \underline{v} \in Q_{l}$, $\underline{v} \neq 0$, such that $\underline{v} \in R_{l}$ and $\underline{v} \in F_{l}$. As $\underline{v} \in F_{l}$ all the moments in $M_{R, l}(\underline{v})$ are equal to zero. But $\underline{v} \in R_{l}$ and $\underline{v} \neq 0$, imply that these moments cannot be zero.

Lemma 15. $F_{l} \subset H\left(\operatorname{curl}^{0}, K\right)$.

Proof. Let $\underline{u} \in F_{l}$. All the moments in $M_{R, l}(\underline{u})$ are equal to zero. This implies by Lemma 8 that $\underline{u}$ is irrotational in $K$.

Lemma 16. $R_{l+1}=Q_{l} \oplus E_{l+1}$.

Proof. Completely analogous to the proof of Lemma 14.

Lemma 17. $\underline{u} \in E_{l+1}$ and $\operatorname{curl} \underline{u}=0$ imply $\underline{u}=0$.

Proof. By Lemma $16 \underline{u} \in R_{l+1}$. Then, by Lemma 9 we have $\underline{u} \in Q_{l}$ and, again by Lemma $16, \underline{u}=0$.

The decompositions provided by Lemmas 14 and 16 are similar to those considered by Nedelec in his original works $[29,30]$ (i.e., $Q_{l}=R_{l} \oplus G_{l}$ and $R_{l+1}=Q_{l} \oplus S_{l+1}$ ) even though the spaces involved are different. Some examples may shed more light on this difference. Let us consider the standard reference tetrahedron and let, on the one hand, $\underline{w}=\operatorname{grad}\left(x-x^{2}-x y-x z\right)=(1-2 x-y-z,-x,-x)$. We have $\underline{w} \in Q_{1}=P_{1}^{3}=R_{1} \oplus G_{1}$ and since $\underline{w} \notin \tilde{P}_{1}^{3} \supset G_{1}$ we obtain $\underline{w} \notin G_{1}$. As the tangential component of $\underline{w}$ along the edge placed on the $\mathrm{x}$ axis is equal to $1-2 x$ and the tangential components along the edges placed on the $\mathrm{y}$ axis or the $\mathrm{z}$ axis are equal to zero all the moments in $M_{R, 1}(\underline{w})$ calculated along these edges are zero. Moreover, since curl $\underline{w}=0$ we conclude that also the moments calculated along the other three edges are equal to zero, i.e., $\underline{w} \in F_{1}$. On the other hand let $\underline{w}^{*}=\operatorname{grad}\left(-x^{2}-x y-x z\right)=(-2 x-y-z,-x,-x) \in G_{1}$. Since the tangential component of $\underline{w}^{*}$ along the edge placed on the $\mathrm{x}$ axis is equal to $-2 x$ it has a nontrivial moment along this edge and, consequently, $\underline{w}^{*} \notin F_{1}$. 
Analogously one can easily verify that $\underline{w}=\left(0,-y z, y^{2}\right) \in S_{2}$ but $\underline{w} \notin E_{2}$ and that $\underline{w}^{*}=\left(0,-y z, y^{2}-y\right) \in E_{2}$ but $\underline{w}^{*} \notin S_{2}$.

An additional important property of the spaces considered is their invariance under the usual affine transformation. To state this property, let the tetrahedron $K$ be affine equivalent to the tetrahedron $\hat{K}$. This means that $[16] \exists F_{K}: \hat{K} \rightarrow K$,

$$
\underline{x}=F_{K}(\underline{\hat{x}})=B_{K} \underline{\hat{x}}+\underline{b}_{K}
$$

( $B_{K}$ invertible) such that

$$
K=F_{K}(\hat{K})
$$

Moreover, suppose that scalar fields defined on $K$ are transformed as follows

$$
\hat{\phi}(\underline{\hat{x}})=\phi\left(F_{K}(\underline{\hat{x}})\right)
$$

while vector fields defined on $K$ are transformed like gradients [20,29]:

$$
\underline{\hat{u}}(\underline{\hat{x}})=B_{K}^{T}\left(\underline{u}\left(F_{K}(\underline{\hat{x}})\right)\right) .
$$

Then we have:

Lemma 18. The spaces $R_{l}, Q_{l}, F_{l}$, and $E_{l+1}, l>0$, are invariant under transformation (27).

Proof. See Appendix A.

Remark 1. On the contrary, one can easily see that $G_{l}$ and $S_{l}$ are not invariant under transformation (27).

In [20], given a triangulation, the edge elements of the family introduced in [29] on tetrahedral meshes are used to define two finite element spaces conforming in $V$ in the two limit cases $\Gamma_{\nu}=\Gamma$ and $\Gamma_{\tau}=\Gamma$. Owing to our assumptions about the triangulation $\mathcal{T}_{h}$, a similar reasoning can be used to define two spaces of finite element vector fields $R_{l, h}$ and $Q_{l, h}$ which are conforming in $V$ in the general case of mixed boundary conditions. As a matter of fact, it is sufficient to consider the spaces $R_{l, h}^{\Gamma=\Gamma_{\nu}}=\left\{\underline{u}_{h} \in H(\operatorname{curl}, \Omega):\left.\underline{u}_{h}\right|_{K} \in R_{l} \forall K \in \mathcal{T}_{h}\right\}$ and $Q_{l, h}^{\Gamma=\Gamma_{\nu}}=\left\{\underline{u}_{h} \in H(\operatorname{curl}, \Omega):\left.\underline{u}_{h}\right|_{K} \in Q_{l} \forall K \in \mathcal{T}_{h}\right\}$ and set the moments of all vector fields of these spaces to zero on all faces constituting $\bar{\Gamma}_{\tau}$ (see Lem. 5.7, Chap. III of [20]). As our assumptions about $\mathcal{T}_{h}$ imply that no element face is shared by $\Gamma_{\tau}$ and $\Gamma_{\nu}$, this is equivalent to define

$$
R_{l, h}=R_{l, h}^{\Gamma=\Gamma_{\nu}} \cap V=\left\{\underline{u}_{h} \in V:\left.\underline{u}_{h}\right|_{K} \in R_{l} \forall K \in \mathcal{T}_{h}\right\}
$$

and

$$
Q_{l, h}=Q_{l, h}^{\Gamma=\Gamma_{\nu}} \cap V=\left\{\underline{u}_{h} \in V:\left.\underline{u}_{h}\right|_{K} \in Q_{l} \forall K \in \mathcal{T}_{h}\right\} .
$$

Remark 2. As a consequence of Lemma 5.7, Chapter III of [20], the standard interpolation operator $r_{l, h}$ : $W^{1, s}(\Omega)^{3} \rightarrow R_{l, h}^{\Gamma=\Gamma_{\nu}}[20]$, with $s>2$, and the analogous one $q_{l, h}: W^{1, s}(\Omega)^{3} \rightarrow Q_{l, h}^{\Gamma=\Gamma_{\nu}}$, are such that $\left(r_{l, h} \underline{u}\right) \times\left.\underline{n}\right|_{\Gamma_{\tau}}=0$ and $\left(q_{l, h} \underline{u}\right) \times\left.\underline{n}\right|_{\Gamma_{\tau}}=0$, whenever $\underline{u} \times\left.\underline{n}\right|_{\Gamma_{\tau}}=0$.

Any vector field $\underline{u} \in R_{l, h}$ (respectively, $Q_{l, h}$ ) is uniquely identified by the set of moments $M_{\Omega, R, l}(\underline{u})=$ $\bigcup_{K \in \mathcal{T}_{h}} M_{R, l}(\underline{u})$ (respectively, $M_{\Omega, Q, l}(\underline{u})=\bigcup_{K \in \mathcal{T}_{h}} M_{Q, l}(\underline{u})$ ) (all the moments on all faces constituting $\bar{\Gamma}_{\tau}$ are set to zero according to (28) and (29)).

Our previous procedure introducing the spaces $F_{l}$ and $E_{l+1}$ can be generalized from $K$ to $\bar{\Omega}$, and leads to the easily defined but fundamental spaces

$$
F_{l, h}=\left\{\underline{u}_{h} \in Q_{l, h}:\left.\underline{u}_{h}\right|_{K} \in F_{l} \forall K \in \mathcal{T}_{h}\right\}
$$


and

$$
E_{l+1, h}=\left\{\underline{u}_{h} \in R_{l+1, h}:\left.\underline{u}_{h}\right|_{K} \in E_{l+1} \forall K \in \mathcal{T}_{h}\right\}
$$

which are curl conforming in $V$. Let us consider $\underline{q} \in Q_{l, h}$ and let $M_{\Omega, Q, l}(\underline{q})$ be the corresponding moments. Moreover, let $\underline{r}(\underline{q})$ be the unique vector field in $R_{l, h}$ such that $M_{\Omega, R, l}(\underline{r}(\underline{q}))=M_{\Omega, R, l}(\underline{q})$. It is then possible to define $\underline{u}_{1}(\underline{q})=\underline{q}-\underline{r}(\underline{q}) \in Q_{l, h} \subset V$. All the moments in $M_{\Omega, R, l}\left(\underline{u}_{1}\right)$ are equal to zero. This happens if and only if all the moments in $M_{R, l}\left(\left.\underline{u}_{1}\right|_{K}\right)$ are equal to zero $\forall K \in \mathcal{T}_{h}$ or, equivalently, $\left.\underline{u}_{1}\right|_{K} \in F_{l} \forall K \in \mathcal{T}_{h}$. Both the linear and everywhere defined operators $\underline{r}(\underline{q}): Q_{l, h} \rightarrow R_{l, h} \subset Q_{l, h}$ and $\underline{u}_{1}(\underline{q}): Q_{l, h} \rightarrow F_{l, h} \subset Q_{l, h}$ are surjective since $\underline{r}\left(R_{l, h}\right)=R_{l, h}$ and $\underline{u}_{1}\left(F_{l, h}\right)=F_{l, h}$. Hence, as $\underline{q}=\underline{r}(\underline{q})+\underline{u}_{1}(\underline{q})$ we obtain $Q_{l, h}=R_{l, h}+F_{l, h}$.

In an analogous way, we can also consider $\underline{r} \in R_{l+1, h}$. If $M_{\Omega, R, l+1}(\underline{r})$ are the moments identifying $\underline{r}$, let $\underline{q}(\underline{r})$ be the unique vector field in $Q_{l, h}$ such that $M_{\Omega, Q, l}(\underline{q}(\underline{r}))=M_{\Omega, Q, l}(\underline{r})$. It is then possible to define $\underline{u}_{2}(\underline{r})=$ $\underline{r}-\underline{q}(\underline{r}) \in R_{l+1, h} \subset V$. All the moments in $M_{\Omega, Q, l}\left(\underline{u}_{2}\right)$ are equal to zero. This happens if and only if all the moments in $M_{Q, l}\left(\left.\underline{u}_{1}\right|_{K}\right)$ are equal to zero $\forall K \in \mathcal{T}_{h}$ or, equivalently, $\left.\underline{u}_{1}\right|_{K} \in E_{l+1} \forall K \in \mathcal{T}_{h}$. Both the linear and everywhere defined operators $\underline{q}(\underline{r}): R_{l+1, h} \rightarrow Q_{l, h} \subset R_{l+1, h}$ and $\underline{u}_{2}(\underline{r}): R_{l, h} \rightarrow E_{l+1, h} \subset R_{l+1, h}$ are surjective since $\underline{q}\left(Q_{l, h}\right)=Q_{l, h}$ and $\underline{u}_{2}\left(E_{l+1, h}\right)=E_{l+1, h}$. Hence, as $\underline{r}=\underline{q}(\underline{r})+\underline{u}_{2}(\underline{r})$ we obtain $R_{l+1, h}=Q_{l, h}+E_{l+1, h}$.

For the spaces $R_{l, h}, Q_{l, h}, F_{l, h}$ and $E_{l+1, h}(l>0)$ we can prove the following crucial properties:

Lemma 19. $Q_{l, h}=R_{l, h} \oplus F_{l, h}$.

Proof. Completely analogous to the proof of Lemma 14.

Lemma 20. $F_{l, h} \subset V_{0}$.

Proof. $\forall \underline{u} \in F_{l, h}$ we know that $\underline{u} \in V$ and $\left.\underline{u}\right|_{K} \in F_{l} \forall K \in \mathcal{T}_{h}$. Then, by Lemma $15, \underline{u}_{1} \in V_{0}$.

Lemma 21. $R_{l+1, h}=Q_{l, h} \oplus E_{l+1, h}$.

Proof. Completely analogous to the proof of Lemma 14.

Lemma 22. $R_{1, h} \subset R_{l, h} \forall l>0$ and $R_{1, h} \subset Q_{l, h} \forall l>0$.

Proof. $R_{1, h} \subset R_{1, h}$ and the thesis follows by using recursively Lemmas 19 and 21 .

\subsection{Nedelec's tetrahedral edge elements are spurious-free}

In this section we prove that $\forall l>0$ both sequences $\left\{R_{l, h}\right\}_{h \in I}$ and $\left\{Q_{l, h}\right\}_{h \in I}$ satisfy (CAS), (CDK) and (DCP) and, therefore, generate spurious-free approximations. This result will be achieved by induction on $l$. In order to do that we firstly have to prove or recall some properties of the sequence $\left\{R_{1, h}\right\}$, which will provide the starting point for the inductive proof. Proofs in this section make use of technical lemmas (numbered from 25 to 36) that can be found in Appendix B.

Theorem 1. $\left\{R_{1, h}\right\}$ satisfies (CAS).

Proof. Let us consider the set $C_{\bar{\Gamma}_{\tau}}^{\infty}(\bar{\Omega})^{3}$ of the vector fields belonging to $C^{\infty}(\bar{\Omega})^{3}$ and vanishing in a neighbourhood of $\bar{\Gamma}_{\tau}$. By Proposition 3.6 of [19], $C_{\bar{\Gamma}_{\tau}}^{\infty}(\bar{\Omega})^{3}$ is dense in $V$. Hence, by applying Theorem 5.4, Chapter III of $[20]$ to $\underline{u} \in C_{\bar{\Gamma}_{\tau}}^{\infty}(\bar{\Omega})^{3}$ and noticing that $r_{1, h} \underline{u} \in R_{1, h}$ (see Rem. 2), by a density argument we obtain that (CAS) holds true.

Let $P_{1, h}$ be the space spanned by the first-order scalar Lagrangian element functions on $\mathcal{T}_{h}$ vanishing on $\bar{\Gamma}_{\tau}$, that is to say $P_{1, h}=\left\{p_{h} \in H_{0, \Gamma_{\tau}}^{1}(\Omega):\left.p_{h}\right|_{K} \in P_{1} \forall K \in \mathcal{T}_{h}\right\}$. As $\operatorname{grad}\left(P_{1}\right) \subset P_{0}^{3} \subset R_{1}$, straightforward application of Lemma 35 with $U_{h}=R_{1, h}$ (see (28)) gives the following two theorems.

Theorem 2. $\operatorname{grad}\left(P_{1, h}\right) \subset R_{1, h}$.

Theorem 3. If $\exists s>1 / 2$ such that $\mathbb{H} \subset H^{s}(\Omega)^{3}$ then $\left\{R_{1, h}\right\}$ satisfies $(C D K)$. 
Moreover, Kikuchi [25] proved the following result:

Theorem 4. If $\exists s>1 / 2$ such that $V_{1}$ and $\mathbb{H} \subset H^{s}(\Omega)^{3}$, and $\exists C>0$ such that $\left\|\underline{v}_{1}\right\|_{s, \Omega} \leq C\left\|\underline{v}_{1}\right\|_{\operatorname{curl}, \Omega} \forall \underline{v}_{1} \in V_{1}$, then $\left\{R_{1, h}\right\}$ satisfies $(D C P)$.

Remark 3. Actually Kikuchi [25] did not consider the case of mixed boundary conditions, but his proof does work even in that case if the hypothesis $\exists s>1 / 2$ such that $V_{1}$ and $\mathbb{H} \subset H^{s}(\Omega)^{3}$, and $\exists C>0$ such that $\left\|\underline{v}_{1}\right\|_{s, \Omega} \leq C\left\|\underline{v}_{1}\right\|_{\text {curl } \Omega} \forall \underline{v}_{1} \in V_{1}$ are still satisfied. These regularity assumptions are not satisfied for general mixed boundary conditions, but are satisfied when mixed boundary conditions arise from symmetry exploitation (see Sect. 2.2). Hence, allowing mixed boundary conditions in Theorem 4 makes sense and is worth-while.

Remark 4. The discrete compactness property considered in [25] is not exactly the same as (DCP) in Theorem 4 , but implies it. In fact, (DCP) in Theorem 4 is a property of sequences $\left\{\underline{x}_{h}\right\}$ with $\underline{x}_{h}$ in the orthogonal complement of $R_{1, h} \cap V_{0}$ to $R_{1, h}$, while in [25] the same property is stated for sequences with $\underline{x}_{h}$ in the orthogonal complement of $\operatorname{grad}\left(P_{1, h}\right)$ to $R_{1, h}$, which is a space containing the former, since $\operatorname{grad}\left(P_{1, h}\right) \subset R_{1, h} \cap V_{0}$.

The following two lemmas are the basic tools to inductively prove (DCP).

Lemma 23. If $\left\{R_{l, h}\right\}_{h \in I}$ satisfies $(C D K)$ and (DCP), then $\left\{Q_{l, h}\right\}_{h \in I}$ satisfies (DCP).

Proof. Direct consequence of Lemmas 19, 20, and 27.

Lemma 24. Let us suppose that $\left\{Q_{l, h}\right\}_{h \in I}$ satisfies (CDK) and (DCP). Moreover, suppose that $\exists s>1 / 2$ such that $V_{1}$ and $\mathbb{H} \subset H^{s}(\Omega)^{3}$ and that $\exists C>0$ such that $\left\|\underline{v}_{1}\right\|_{s, \Omega} \leq C\left\|\underline{v}_{1}\right\|_{\text {curl }, \Omega} \forall \underline{v}_{1} \in V_{1}$. Then $\left\{R_{l+1, h}\right\}_{h \in I}$ satisfies $(D C P)$.

Proof. By Lemma $21 R_{l+1, h}=Q_{l, h} \oplus E_{l+1, h}$. Note that $\forall \underline{w}_{h} \in E_{l+1, h}$ we have $\left.\underline{w}_{h}\right|_{K} \in E_{l+1} \forall K \in \mathcal{T}_{h} \forall h \in I$ and that by Lemma 18 the space $E_{l+1}$ is invariant under transformation (27). But by Lemma 17 if $\left.\underline{w}_{h}\right|_{K} \in E_{l+1}$ and curl $\left.\underline{w}_{h}\right|_{K}=0$ we have $\left.\underline{w}_{h}\right|_{K}=0$. Then the hypotheses of Corollary 2 are satisfied and, consequently, $\exists C_{1}>0, C_{1}$ independent of $\underline{w}_{h}$ and $h$ such that $\left\|\left.\underline{w}_{h}\right|_{K}\right\|_{1, K} \leq C_{1}\left\|\left.\underline{w}_{h}\right|_{K}\right\|_{\text {curl,K}} \forall k \in \mathcal{T}_{h}$ and $\forall \underline{w}_{h} \in E_{l+1, h}, i . e .$, one of the hypotheses of Lemma 36 is satisfied. Moreover, $\forall \underline{v}_{h} \in R_{l+1, h}$ we have $\underline{v}_{h}=\underline{u}_{h}+\underline{w}_{h}$, where $\underline{u}_{h} \in Q_{l, h}$ and $\underline{w}_{h} \in E_{l+1, h}$, and $\left.\underline{v}_{h}\right|_{K} \in R_{l+1},\left.\underline{u}_{h}\right|_{K} \in Q_{l}$, and $\left.\underline{w}_{h}\right|_{K} \in E_{l+1}, \forall K \in \mathcal{T}_{h} \forall h \in I$. Again by Lemma $18 R_{l+1}$, $Q_{l}$, and $E_{l+1}$ are invariant under transformation (27). Then the hypotheses of Lemma 31 are satisfied. Consider also that, by Lemma 9 , any vector field $\underline{\hat{z}}=\underline{\hat{u}}+\underline{\hat{y}}, \underline{\hat{z}} \in R_{l+1}, \underline{\hat{x}} \in Q_{l}, \underline{\hat{y}} \in E_{l+1}$, satisfying curl $\underline{\hat{z}}=0$ actually belongs to $Q_{l}$, i.e., $\hat{y}=0$. Then also the hypotheses of Lemma 32 are satisfied. The conclusions of Lemmas 31 and 32 implies that the hypotheses of Lemma 34 are satisfied. Consequently its conclusions hold true as well, i.e., $\exists \exists C_{2}, C_{3}>0, C_{2}$ and $C_{3}$ independent of $\left\{\underline{v}_{h}\right\}$, such that $\left\|\underline{u}_{h}\right\|_{\text {curl }, \Omega} \leq C_{2}$ and $\left\|\underline{w}_{h}\right\|_{\text {curl }, \Omega} \leq C_{3}$. This means that another hypothesis of Lemma 36 is satisfied. Finally, by Theorem 2 and Lemma $22, \operatorname{grad}\left(P_{1, h}\right) \subset Q_{l, h}$ $\forall l>0, \forall h \in I$. As all the hypotheses of Lemma 36 are satisfied its conclusion holds true and this conclude the proof.

Finally, we are now ready to work out the inductive procedure and prove the following theorem, which is crucial to show the spurious-free character of Nedelec's tetrahedral edge elements.

Theorem 5. Let us suppose that $\exists s>1 / 2$ such that $V_{1}$ and $\mathbb{H} \subset H^{s}(\Omega)^{3}$, that $\exists C>0$ such that $\left\|\underline{v}_{1}\right\|_{s, \Omega} \leq$ $C\left\|\underline{v}_{1}\right\|_{\text {curl }, \Omega} \forall \underline{v}_{1} \in V_{1}$. Then $\left\{R_{l, h}\right\}_{h \in I}$ and $\left\{Q_{l, h}\right\}_{h \in I}$ satisfy $(C A S)$, (CDK), and (DCP) $\forall l>0$.

Proof. By Theorems 1, 3 and 4 we know that $\left\{R_{1, h}\right\}$ satisfies the three conditions. Moreover (Lem. 22) $R_{1, h} \subset R_{l, h}$ and $R_{1, h} \subset Q_{l, h} \forall l>0$ and $\forall h \in I$. Then, by Lemmas 25 and 26 we have that both sequences satisfy (CAS) and (CDK) $\forall l>0$. Moreover, starting from Theorem 4, we can inductively use Lemmas 23 and 24 to conclude the proof.

It is now possible to prove our main results on Nedelec's elements, which are very important from a practical viewpoint and, for the sake of clarity, will be stated with explicit reference to the hypotheses on $\Omega, \Gamma_{\tau} \Gamma_{\nu}$, $\mu, \epsilon$ and $\left\{\mathcal{T}_{h}\right\}$, which have been often understood in previous theorems, after stating them once for all at the beginning. 
Theorem 6. Under the hypotheses assumed in Section 2.1 in defining Problem 1 and in Section 3 in defining Problem 4, the space sequences $\left\{R_{l, h}\right\}_{h \in I}$ and $\left\{Q_{l, h}\right\}_{h \in I}$ satisfy (CAS), (CDK) and (DCP) $\forall l>0$.

Proof. The problem we are approximating by the finite element method is Problem 1. To this problem we can associate Problem 2 as indicated in Section 2.2. If mixed boundary conditions are introduced only to exploit possible symmetries of the actual cavity resonator, by Lemmas 3,4 , and 5 we obtain that $\exists s>1 / 2$ such that $V_{1, p b 2}$ and $\mathbb{H}_{p b 2} \subset H^{s}(\Omega)^{3}$, that $\exists C>0$ such that $\left\|\underline{v}_{1}\right\|_{s, \Omega} \leq C\left\|\underline{v}_{1}\right\|_{\text {curl }, \Omega} \forall \underline{v}_{1} \in V_{1, p b 2}$. Then by Theorem 5 $\left\{R_{l, h}\right\}_{h \in I}$ and $\left\{Q_{l, h}\right\}_{h \in I}$ satisfy (CAS), (CDK) and (DCP) $\forall l>0$ for Problem 2, i.e., for $\epsilon=1=\mu$. But by using Propositions 2.25, 2.26, and 2.27 of [13] we can conclude that the space sequences $\left\{R_{l, h}\right\}_{h \in I}$ and $\left\{Q_{l, h}\right\}_{h \in I}$ satisfy (CAS), (CDK) and (DCP) $\forall l>0$ for the tensor fields $\epsilon$ and $\mu$ of actual interest, i.e., those involved in Problem 1.

Theorem 7. Under the hypotheses assumed in Section 2.1 in defining Problem 1 and in Section 3 in defining Problem 4, if either $V_{h}=R_{l, h}$ or $V_{h}=Q_{l, h}$, then $\forall l>0$ Problem 4 is a spurious-free approximation of Problem 6, in the sense of [13].

Proof. Direct consequence of Theorem 6 and of Theorem 6.9 of [13].

Remark 5. The hypotheses of Theorems 6 and 7 permit multiply connected problem domains with nonconnected boundary, inhomogeneous, anisotropic and discontinuous material properties and mixed boundary conditions introduced to exploit symmetries, so covering almost all situations of practical interest.

\subsection{Construction of new families of spurious-free elements}

It is well known that Nedelec's edge elements of the first family [29] provide a good balance in the accuracy of representation of the field and its curl [34] and that, on the contrary, Nedelec's edge elements of the second family [30] provide a better accuracy of the field than its curl [34]. Elements of both families have been widely used and even though the debate on which family provides better results has never come to an end it is now clear that either one family or the other may be the best one depending on the considered problem [34]. Hence, even a family of edge elements providing better approximations of the curl of a field than of the field itself may be useful in specific cases. In fact, the elements of such a family would be expected to provide better results than the standard Nedelec's elements $[29,30]$ in problems where the curl of the field is more important than the field itself.

On the other hand, one might still think that such a new element is useless on the basis of the following consideration. If the problem were originally formulated in terms of the electric field (magnetic field), in principle, the performances could be improved also by recasting it in terms of the magnetic field (electric field) and by using the elements of the second Nedelec's family [30] which better approximate the field than its curl. However, such a switch in the problem formulation may not be so convenient from a practical point of view and this fact makes worth developing the hypothetical new family, which would complete in some sense the previously defined Nedelec's families. As a matter of fact, if this family were available, it would be possible to define a single algorithm making use of the best element in all situations.

The target of this section is then to define this new family of edge elements by using the already developed tools. First of all let us consider that $Q_{l}=R_{l} \oplus F_{l}$ and that the space $F_{l}$, being made of irrotational fields, is used only to complete (to the order $l$ ) the polynomial order of the field representation. Secondly $R_{l+1}=$ $Q_{l} \oplus E_{l+1}=R_{l} \oplus F_{l} \oplus E_{l+1}$ and $E_{l+1}$ is used to complete (to the order $l$ ) the polynomial order of the curl of the field but not of the field itself (to the order $l+1$ ). We can then quite naturally define a new space of polynomial vector fields $P_{1, l}=R_{l} \oplus E_{l+1}, l>0$, whose curl, being $F_{l}$ irrotational, is the same as that of $R_{l+1}$, i.e., $\operatorname{curl}\left(P_{1, l}\right)=\operatorname{curl}\left(R_{l+1}\right)$. The polynomial order of approximation, however, is not even complete to the order $l$ but only to the order $l-1$. These are the feature we were looking for. However, in order to completely define a new finite element we must also provide a set of $P_{1, l}$-unisolvent [16] linear forms defined over the space $P_{1, l}$. Let us consider as set of linear forms the following set of moments $\left(\underline{u} \in P_{1, l}\right)$

$$
M_{R, l}(\underline{u}) \cup\left(M_{R, l+1}(\underline{u}) \backslash M_{Q, l}(\underline{u})\right)
$$


and remember that (with a suitable choice of the basis) $M_{R, l}(\underline{u}) \subset M_{Q, l}(\underline{u}) \subset M_{R, l+1}(\underline{u})$. As $\operatorname{dim}\left(R_{l}\right)=$ $\operatorname{card}\left(M_{R, l}(\underline{u})\right)$ and $\operatorname{dim}\left(E_{l+1}\right)=\operatorname{card}\left(M_{R, l+1}(\underline{u}) \backslash M_{Q, l}(\underline{u})\right)$ we easily conclude that $\operatorname{dim}\left(P_{1, l}\right)=\operatorname{card}\left(M_{R, l}(\underline{u}) \cup\right.$ $\left.\left(M_{R, l+1}(\underline{u}) \backslash M_{Q, l}(\underline{u})\right)\right)$. Moreover, let us suppose that one nonzero function $\underline{u} \in P_{1, l}$ has all the moments in $M_{R, l}(\underline{u}) \cup\left(M_{R, l+1}(\underline{u}) \backslash M_{Q, l}(\underline{u})\right)$ equal to zero. Since $\underline{u}=\underline{v}+\underline{w}, \underline{v} \in R_{l}$ and $\underline{w} \in E_{l+1}$ and due to the fact that all the moments in $M_{R, l}(\underline{w}) \subset M_{Q, l}(\underline{w})$ are equal to zero we have $\underline{v}=0$. Then $\underline{u}=\underline{w}$ and since all moments in $M_{R, l+1}(\underline{u}) \backslash M_{Q, l}(\underline{u})$ are zero we have $\underline{u}=\underline{w}=0$ and the $P_{1, l}$-unisolvence is established.

Analogously to what we did in the previous Section for the standard Nedelec's spaces, we may now define the spaces (defined on $\bar{\Omega}$ ) $P_{1, l, h}=R_{l, h} \oplus E_{l+1, h}$ (see equations (28), (31)).

Being the definition of the new family of edge elements complete we have still to address the question of its capability to produce spurious-free approximations of electromagnetic eigenproblems. But by Lemmas 25 and 26 the space sequence $\left\{P_{1, l, h}\right\}, l>0$, satisfies (CAS) and (CDK) when $\left\{R_{l, h}\right\}$ does and with obvious minor modification in the statement of Lemma 24 they also satisfy (DCP) $\forall l>0$ (in the proof one has to change only one part which uses Lemma 9 and argue as follows: if $\underline{\hat{z}}=\underline{\hat{u}}+\hat{\hat{y}}, \underline{\hat{z}} \in P_{1, l} \subset R_{l+1}, \underline{\hat{u}} \in R_{l} \subset Q_{l}, \hat{y} \in E_{l+1}$, satisfies curl $\underline{\hat{z}}=0$, we have by Lemma 9 that $\underline{\hat{z}} \in Q_{l}$, and then also $\underline{\hat{y}} \in Q_{l}$. As, by Lemma $16, E_{l+1} \cap Q_{l}=\{0\}$ we obtain $\underline{\hat{y}}=0)$. Thus for the space sequences $\left\{P_{1, l, h}\right\}_{h \in I}, l>0$, we could state theorems analogous to Theorems $\overline{6}$ and 7 .

Remark 6. One may note that it is possible to generalize the previous definition by considering the following spaces $P_{m, l}=R_{l} \oplus E_{l+1} \oplus \cdots \oplus E_{l+m}, l>0$, and as sets of moments those belonging to $M_{R, l} \cup\left(M_{R, l+1} \backslash M_{Q, l}\right) \cup$ $\cdots \cup\left(M_{R, l+m} \backslash M_{Q, l+m-1}\right)$.

The new family of edge elements just introduced may be considered as a particular application of the following procedure which may be used to systematically construct by induction new families of spurious-free elements starting from an element having the same property.

Let us suppose that, on the one hand, we have to solve a problem characterized by $V_{1}$ and $\mathbb{H} \subset H^{s}(\Omega)^{3}$, for some $s>1 / 2$. By using the Lemmas proved in Section 2.2 and thanks to the results proved in [13] these are not restrictive hypotheses. As a matter of fact, all practical problems defined on topologically complicated domains and involving discontinuous material properties may be dealt with and symmetries may be exploited to reduce the computational cost. On the other hand, let $\left\{U_{h}\right\}$ be a sequence of affine finite element spaces satisfying (CAS), (CDK) and (DCP) (note that (CAS) is a classical property, (CDK) may be verified by exploiting Lemma 35 and (DCP) may be proved by using some established techniques $[4,5,25,28])$. Suppose in particular that $U_{h}=\left\{\underline{u}_{h} \in V:\left.\underline{u}_{h}\right|_{K} \in U \forall K \in \mathcal{T}_{h}\right\}$, where $U \subset H$ (curl, $K$ ) is invariant under transformation (27) and such that $\operatorname{grad}\left(P_{1}\right) \subset U$. If new sequences $\left\{V_{h}\right\}$ of finite element spaces are generated from $\left\{U_{h}\right\}$ in such a way that $U_{h} \subset V_{h} \forall h \in I$ properties (CAS) and (CDK) are preserved (by Lems. 25 and 26) and obviously also the local property concerning the gradient of $P_{1}$ is retained. However, (DCP) may be lost.

Fortunately this does not happen when $V_{h}$ is obtained by sum of $U_{h}$ with an irrotational space $W_{h}$ (Lem. 27) or when the added space, necessarily made up of non-irrotational vector fields, satisfies the hypotheses of Lemma 36 (see also Rem. 7). Note, however, that these last two Lemmas may also be useful when $W_{h}$ can be thought as the sum of an irrotational subspace and a non-irrotational subspace satisfying again the hypotheses of Lemma 36.

It is important to note that the family so constructed is not uniquely determined by the element used at the beginning of the inductive procedure. This implies, in particular, that it is possible to look for the best element, in terms of performances, among those spurious-free elements which can be constructed starting from the same element.

\section{Conclusions}

In this paper we use a new inductive approach to prove, under realistic assumptions, that all the elements of both Nedelec's edge element families defined on tetrahedral meshes guarantee the convergence of the Galerkin finite element approximations of electromagnetic eigenproblems modelling cavity resonators (for the sake of preciseness, they are "spurious-free" in the sense of [13]). Moreover, the tools developed to work out the above 
proof make it possible to systematically define new families of spurious-free finite elements and are, in fact, used to define a new family of elements, alternative to Nedelec's ones, which may be convenient in particular cases.

\section{Appendix A \\ Proof of Lemma 18}

By Lemma 5.5, Chapter III of [20] $R_{l}$ is invariant. In the proof of the same lemma also $Q_{l}$ is proved to be invariant. As for $F_{l}$, Lemma 5.6, Chapter III of [20] proves that for any $\underline{u} \in W^{1, s}(K)(s>2)$ if all the moments in $M_{R, l}(\underline{u})$ are equal to zero then all the moments in $M_{R, l}(\underline{\hat{u}})$ are equal to zero, where $M_{R, l}(\underline{\hat{u}})$ is the set of moments given by (22) but defined on the reference tetrahedron $\hat{K}$ and $\underline{\hat{u}}$ is obtained from $\underline{u}$ by (27). Moreover, given $\underline{u} \in F_{l} \subset Q_{l}$ on $K$ we obtain from the invariance of $Q_{l}$ that $\underline{\hat{u}} \in Q_{l}$ on $\hat{K}$. Then, by Definition $1, \underline{\hat{u}} \in F_{l}$ on $\hat{K}$, i.e., $F_{l}$ is invariant as well. The proof concerning $E_{l+1}$ is quite similar. First of all note that $E_{l+1} \subset R_{l+1}$ and that we have already proved that $R_{l+1}$ is invariant under transformation (27). Thus, to conclude the proof we have to show that given $\underline{u} \in R_{l+1}$ on $K$ and such that all the moments in $M_{Q, l}(\underline{u})$ are equal to zero the vector field $\underline{\hat{u}}$, obtained from $\underline{u}$ by $(27)$, is such that all the moments in $M_{Q, l}(\underline{\hat{\imath}})$ are equal to zero, where $M_{Q, l}(\underline{\hat{\imath}})$ is the set of moments given by (23) but defined on the reference tetrahedron $\hat{K}$. This is worked out in three steps. Let $\underline{u} \in E_{l+1}$. Firstly, let us prove that all the moments in $M_{E, Q, l}(\underline{u})$ are equal to zero if and only if all the moments in $M_{E, Q, l}(\underline{\hat{u}})$ are. Observe that $M_{E, Q, l}(\underline{u})=M_{E, R, l+1}(\underline{u})$. Moreover, from Lemma 5.6, Chapter III of [20], we have that all the moments in $M_{E, R, l+1}(\underline{u})$ are equal to zero if and only if all the moments in $M_{E, R, l+1}(\underline{\hat{u}})$ are. Then, this first conclusion immediately follows. Secondly, let us prove that all the moments in $M_{K, Q, l}(\underline{u})$ are equal to zero if and only if all the moments in $M_{K, Q, l}(\underline{\hat{u}})$ are. From the proof of Lemma 5.6, Chapter III of [20], we deduce

$$
\int_{K} \underline{u} \cdot \underline{q}=\left|\operatorname{det} B_{K}\right| \int_{\hat{K}} \underline{\hat{u}} \cdot\left(B_{K}^{-1}\right)\left(\underline{q}\left(F_{K}(\underline{\hat{x}})\right)\right) .
$$

But thanks to Proposition 5.2, Chapter III of [20] we have that the vector field

$$
\underline{\hat{q}}=\left(B_{K}^{-1}\right)\left(\underline{q}\left(F_{K}(\underline{\hat{x}})\right)\right) \in D_{l-2}(\hat{K}) \quad \forall \underline{q} \in D_{l-2}(K) .
$$

Then,

$$
\int_{K} \underline{u} \cdot \underline{q}=\left|\operatorname{det} B_{K}\right| \int_{\hat{K}} \underline{\hat{u}} \cdot \underline{\hat{q}}
$$

and the second conclusion follows because, as $\left|\operatorname{det} B_{K}\right| \neq 0,(34)$ implies that

$$
\int_{K} \underline{u} \cdot \underline{q}=0 \forall \underline{q} \in D_{l-2}(K) \Longleftrightarrow \int_{\hat{K}} \underline{\hat{u}} \cdot \underline{\hat{q}}=0 \forall \underline{\hat{q}} \in D_{l-2}(\hat{K}) .
$$

Finally, let us prove that all the moments in $M_{F, Q, l}(\underline{u})$ are equal to zero if and only if all the moments in $M_{F, Q, l}(\underline{\hat{u}})$ are. The moments we are now considering are defined and easily computed in a $2 \mathrm{D}$ Cartesian reference system on $f$ that, for the present purpose, we complete with a third axis orthogonal to $f$. Let us denote by ' the quantities in this new 3D local reference system. It is obvious that we can extend any element of $D_{l-1}(f)$ to a three-dimensional vector field $\underline{q}^{\prime} \in D_{l-1}(K)$ having that element as tangential component on $f$ and such that $\underline{q}^{\prime} \cdot \underline{n}^{\prime}=0$ on $f$. Conversely, the restriction to $f$ of any $\underline{q}^{\prime} \in D_{l-1}(K)$ such that $\underline{q}^{\prime} \cdot \underline{n}^{\prime}=0$ on $f$ belongs to $D_{l-1}(f)$. Hence, the moments under consideration can be computed as $\int_{f} \underline{u}^{\prime} \cdot \underline{q}^{\prime}$. By using the same procedure the moments $M_{F, Q, l}(\underline{\hat{u}})$ can be computed as $\int_{\hat{f}} \underline{\underline{u}}^{\prime} \cdot \underline{\underline{q}}^{\prime}$, where ${ }^{\prime}$ denotes the quantities in a 3D reference system local to $\hat{f}$ (obtained as before by adding a third axis orthogonal to a 2D Cartesian reference system on $\hat{f}$ ), and $\underline{\hat{q}}^{\prime} \in D_{l-1}(\hat{K})$ is such that $\underline{\hat{q}}^{\prime} \cdot \underline{\hat{n}}^{\prime}=0$ on $\hat{f}$. 
Since $\underline{x}$ and $\underline{x}^{\prime}$ are linked by an orthogonal transformation, we have simply

$$
\int_{f} \underline{u}^{\prime} \cdot \underline{q}^{\prime}=\int_{f} \underline{u} \cdot \underline{q}
$$

with $\underline{q} \in D_{l-1}(K)$ and $\underline{q} \cdot \underline{n}=0$ on $f$.

Analogously, since also $\underline{\hat{x}}$ and $\underline{\hat{x}}^{\prime}$ are linked by an orthogonal transformation, we obtain

$$
\int_{\hat{f}} \underline{\hat{u}}^{\prime} \cdot \underline{\hat{q}}^{\prime}=\int_{\hat{f}} \underline{\hat{u}} \cdot \underline{\hat{q}}
$$

with $\underline{\hat{q}} \in D_{l-1}(\hat{K})$ and $\underline{\hat{q}} \cdot \underline{\hat{n}}=0$ on $\hat{f}$.

Let us now consider the usual affine transformation (24) from $\underline{\hat{x}}$ to $\underline{x}$. Then, exactly in the same way as we deduced equation (34),

$$
\int_{f} \underline{u} \cdot \underline{q}=\left|\operatorname{det} B_{K}\right| \int_{\hat{f}} \underline{\hat{u}} \cdot \underline{\hat{q}}
$$

where $\underline{\hat{q}} \in D_{l-1}(\hat{K})$. Moreover,

$$
0=\underline{q}(\underline{x}) \cdot \underline{n}(\underline{x})=\underline{q}\left(F_{K}(\underline{\hat{x}})\right) \cdot \underline{n}\left(F_{K}(\underline{\hat{x}})\right)=B_{K} \underline{\hat{q}}(\underline{\hat{x}}) \cdot \underline{n}\left(F_{K}(\underline{\hat{x}})\right)=\underline{\hat{q}}(\underline{\hat{x}}) \cdot B_{K}^{T} \underline{n}\left(F_{K}(\underline{\hat{x}})\right)
$$

and by using also equation (5.27), p. 265 of [20],

$$
0=\underline{q}(\underline{x}) \cdot \underline{n}(\underline{x})=\frac{1}{\left\|\left(B_{K}^{-1}\right)^{T} \cdot \underline{\hat{n}}\right\|} \underline{\hat{q}}(\underline{\hat{x}}) \cdot \underline{\hat{n}}(\underline{\hat{x}}) .
$$

As $\left|\operatorname{det} B_{K}\right| \neq 0$, from $(38)$ and $(40)$ we deduce

$$
\int_{f} \underline{u} \cdot \underline{q}=0 \forall \underline{q} \in D_{l-1}(K): \underline{q} \cdot \underline{n}=0 \text { on } f \Longleftrightarrow \int_{\hat{f}} \underline{\hat{u}} \cdot \underline{\hat{q}}=0 \forall \underline{\hat{q}} \in D_{l-1}(\hat{K}): \underline{\hat{q}} \cdot \underline{\hat{n}}=0 \text { on } \hat{f}
$$

and the conclusion follows by using (36) and (37).

\section{ApPENDIX B}

\section{Technical lemmas used in the proofs of Section 3.3}

In this appendix we develop some technical lemmas which are used in the proofs leading to our main results on Nedelec's elements. Some of them are reported just for reference but many others need to be fully developed.

Let us start with three lemmas used in building the inductive procedure.

Lemma 25. If $U_{h} \subset V_{h} \subset V \forall h \in I$ and $\left\{U_{h}\right\}$ satisfies (CAS) then $\left\{V_{h}\right\}$ satisfies (CAS).

Lemma 26. If $U_{h} \subset V_{h} \subset V \forall h \in I$ and $\left\{U_{h}\right\}$ satisfies (CDK) then $\left\{V_{h}\right\}$ satisfies (CDK).

Lemma 27. If $V \supset V_{h}=U_{h}+W_{h} \forall h \in I,\left\{U_{h}\right\}$ satisfies (CDK) and (DCP) and $W_{h} \subset V_{0} \forall h \in I$ then $\left\{V_{h}\right\}$ satisfies (DCP) and the limit $\underline{v}$ in $(D C P)$ actually belongs to $V_{1}$.

Proof. Let us consider a sequence $\left\{\underline{v}_{h}\right\}$ such that $\underline{v}_{h} \in V_{1 h},\left\|\underline{v}_{h}\right\|_{\text {curl }, \Omega} \leq 1 \forall h \in I$. As $V_{h}=U_{h}+W_{h}$ we have that $\underline{v}_{h}=\underline{u}_{h}+\underline{w}_{h}, \underline{u}_{h} \in U_{h}, \underline{w}_{h} \in W_{h}, \forall h \in I$. But $W_{h} \subset V_{0}$ means that curl $\underline{w}_{h}=0$. Then $\left\|\operatorname{curl} \underline{v}_{h}\right\|_{0, \Omega}=$ $\left\|\operatorname{curl} \underline{u}_{h}\right\|_{0, \Omega}$ and since $\|\underline{u}\|_{\operatorname{curl}, \Omega} \geq\|\operatorname{curl} \underline{u}\|_{0, \Omega} \underline{\forall} \underline{u} \in V$ we obtain $1 \geq\left\|\underline{v}_{h}\right\|_{\text {curl }, \Omega} \geq\left\|\operatorname{curl} \underline{v}_{h}\right\|_{0, \Omega}=\left\|\operatorname{curl} \underline{u}_{h}\right\|_{0, \Omega}$. Moreover, observe that it is always possible to write $\underline{u}_{h}=\underline{u}_{1 h}+\underline{u}_{0 h}, \underline{u}_{0 h} \in U_{0 h}=U_{h} \cap V_{0}, \underline{u}_{1 h} \in U_{1 h}, U_{1 h}$ 
being the orthogonal complement of $U_{0 h}$ in $U_{h}$. Then, as curl $\underline{u}_{h}=\operatorname{curl} \underline{u}_{1 h}$ we have $1 \geq\left\|\operatorname{curl} \underline{u}_{1 h}\right\|_{0, \Omega}$. But by Corollary 2.20 of [13] $\exists \alpha \geq 0:\left\|\operatorname{curl} \underline{u}_{1 h}\right\|_{0, \Omega}^{2} \geq \alpha\left\|\underline{u}_{1 h}\right\|_{\text {curl }, \Omega}^{2}$. Consequently, $1 / \sqrt{\alpha} \geq\left\|\underline{u}_{1 h}\right\|_{\text {curl }, \Omega}$. Now let us consider $\underline{u}_{0 h}+\underline{w}_{h}=\underline{x}_{h} \in V_{0 h}$ and note that $\left\|\underline{x}_{h}\right\|_{\mathrm{curl}, \Omega}=\left\|\underline{v}_{h}-\underline{u}_{1 h}\right\|_{\mathrm{curl}, \Omega} \leq 1+1 / \sqrt{\alpha}$. Since $\underline{v}_{h} \in V_{1 h}$ and $\underline{x}_{h} \in V_{0 h}$ we have $\left(\underline{v}_{h}, \underline{x}_{h}\right)_{0, \Omega}=0=\left(\underline{u}_{1 h}, \underline{x}_{h}\right)_{0, \Omega}+\left(\underline{x}_{h}, \underline{x}_{h}\right)_{0, \Omega} \forall h \in I$. As $\left\|\underline{u}_{1 h}\right\|_{\text {curl, }, \Omega}$ is bounded, by using (DCP) and Proposition 2.18 of [13], $\underline{u}_{1 h} \rightarrow \underline{u} \in V_{1}$ strongly in $H$ up to a subsequence, as $h \rightarrow 0$. As $\left\|\underline{x}_{h}\right\|_{0, \Omega}$ is bounded and $\underline{x}_{h} \in V_{0 h} \subset V_{0}, \underline{x}_{h} \rightarrow \underline{x} \in V_{0}$ weakly in $H$ up to a subsequence. Hence, again up to a subsequence, $\left(\underline{u}_{1 h}, \underline{x}_{h}\right)_{0, \Omega} \rightarrow(\underline{u}, \underline{x})_{0, \Omega}=0$ and, then, $\left\|\underline{x}_{h}\right\|_{0, \Omega} \rightarrow 0$. As $\underline{v}_{h}=\underline{u}_{1 h}+\underline{x}_{h}$ we obtain $\underline{v}_{h} \rightarrow \underline{u} \in V_{1}$ strongly in $H$ up to a subsequence.

In the following we report some bounds on the norms or seminorms of some vector fields. To this aim, let us suppose that for a given $h$, the tetrahedron $K \in \mathcal{T}_{h}$ is affine equivalent to a fixed tetrahedron $\hat{K}$ in the sense of (24) and (25), and that vector fields defined on $K$ are transformed by (27). It is then possible to show that $[16,20]$ :

$$
\begin{aligned}
|\underline{u}|_{1, K} & \leq C_{42}\left\|B_{K}^{-1}\right\|^{2}\left|\operatorname{det} B_{K}\right|^{1 / 2}|\hat{\hat{u}}|_{1, \hat{K}} \\
\|\operatorname{curl} \underline{\hat{u}}\|_{0, \hat{K}} & \leq C_{43}\left\|B_{K}\right\|^{2}\left|\operatorname{det} B_{K}^{-1}\right|^{1 / 2}\|\operatorname{curl} \underline{u}\|_{0, K} \\
\|\underline{u}\|_{0, K} & \geq C_{44}\left\|B_{K}\right\|^{-1}\left|\operatorname{det} B_{K}\right|^{1 / 2}\|\underline{\hat{u}}\|_{0, \hat{K}} \\
\|\underline{\hat{u}}\|_{0, \hat{K}} & \geq C_{45}\left\|B_{K}^{-1}\right\|^{-1}\left|\operatorname{det} B_{K}^{-1}\right|^{1 / 2}\|\underline{u}\|_{0, K} \\
\|\operatorname{curl} \underline{u}\|_{0, K} & \geq C_{46}\left\|B_{K}\right\|^{-2}\left|\operatorname{det} B_{K}\right|^{1 / 2}\|\operatorname{curl} \underline{\hat{u}}\|_{0, \hat{K}} \\
\|\operatorname{curl} \underline{\hat{u}}\|_{0, \hat{K}} & \geq C_{47}\left\|B_{K}^{-1}\right\|^{-2}\left|\operatorname{det} B_{K}^{-1}\right|^{1 / 2}\|\operatorname{curl} \underline{u}\|_{0, K} .
\end{aligned}
$$

If $h_{K}$ is the diameter of $K$ and $\rho_{K}$ is the supremum of the diameters of the spheres inscribed in $K$, we have [16]

$$
\begin{aligned}
\left\|B_{K}\right\| & \leq h_{K} / \rho_{\hat{K}} \\
\left\|B_{K}^{-1}\right\| & \leq h_{\hat{K}} / \rho_{K} .
\end{aligned}
$$

Other less obvious bounds need to be proved. These bounds are firstly deduced on $\hat{K}$ and then on $K, K \in \mathcal{T}_{h}$, $h \in I$.

Lemma 28. If $Z$ is a finite dimensional subspace of $L^{2}(\hat{K})^{3}$ such that $Z=X \oplus Y$, then for all $\underline{\hat{z}} \in Z$, $\underline{\hat{z}}=\underline{\hat{\hat{x}}}+\underline{\hat{y}}$, $\underline{\hat{x}} \in X, \underline{\hat{y}} \in Y, \exists C>0, C$ independent of $\underline{\hat{z}}$, such that

$$
\|\underline{\hat{z}}\|_{0, \hat{K}} \geq C\|\underline{\hat{y}}\|_{0, \hat{K}} .
$$

Proof. (50) is satisfied if $\underline{\hat{y}}=0$. Let us suppose then that $\underline{\hat{y}} \neq 0$ and define

$$
\mathcal{F}(\underline{\hat{y}})=\min _{\underline{\hat{x}} \in X}\|\underline{\hat{x}}+\underline{\hat{y}}\|_{0, \hat{K}}=\left\|\underline{\hat{y}}-P_{X}(\underline{\hat{y}})\right\|_{0, \hat{K}}
$$

where $P_{X}$ is the projection operator from $Y$ into $X$, orthogonal with respect to $(,)_{0, \hat{K}}$. From $X \cap Y=\{0\}$ it follows that $\mathcal{F}(\underline{\hat{y}}) \neq 0 \forall \underline{\hat{y}} \neq 0$. Moreover $P_{X}$ is continuous and, consequently, $\mathcal{F}$ is a continuous functional on $Y-\{0\}$. Consider $\underline{\hat{y}}^{*}=\underline{\hat{y}} /\|\underline{\hat{y}}\|_{0, \hat{K}}$. Since the surface of the unit sphere in $Y$ is a compact set as it is a bounded and closed subset of a finite dimensional space, there exists $C>0$ such that

$$
\min _{0 \neq \underline{\hat{y}} \in Y} \mathcal{F}\left(\underline{\hat{y}}^{*}\right)=\min _{\substack{\hat{y} \in Y \\\|\underline{\hat{y}}\|_{0, \hat{K}}=1}} \mathcal{F}(\underline{\hat{y}})=C .
$$


Moreover,

$$
\mathcal{F}(\underline{\hat{y}})=\left\|\underline{\hat{y}}-P_{X}(\underline{\hat{y}})\right\|_{0, \hat{K}}=\|\underline{\hat{y}}\|_{0, \hat{K}}\left\|\underline{\hat{y}}^{*}-P_{X}\left(\underline{\hat{y}}^{*}\right)\right\|_{0, \hat{K}}=\|\underline{\hat{y}}\|_{0, \hat{K}} \mathcal{F}\left(\underline{\hat{y}}^{*}\right) \geq C\|\underline{\hat{y}}\|_{0, \hat{K}} .
$$

Then we have

$$
\|\underline{\hat{z}}\|_{0, \hat{K}} \geq \min _{\underline{\hat{x}} \in X}\|\underline{\hat{x}}+\underline{\hat{y}}\|_{0, \hat{K}}=\mathcal{F}(\underline{\hat{y}}) \geq C\|\underline{\hat{y}}\|_{0, \hat{K}}
$$

Lemma 29. If $Z$ is a finite dimensional subspace of $H(\operatorname{curl}, \hat{K})$ such that $Z=X \oplus Y$ and for all $\underline{\hat{z}} \in Z$, $\underline{\hat{z}}=\underline{\hat{x}}+\underline{\hat{y}}, \underline{\hat{x}} \in X, \underline{\hat{y}} \in Y, \operatorname{curl} \underline{\hat{z}}=0$ implies $\underline{\hat{y}}=0$, then $\exists C>0, C$ independent of $\underline{\hat{z}}$, such that

$$
\|\operatorname{curl} \underline{\hat{z}}\|_{0, \hat{K}} \geq C\|\operatorname{curl} \underline{\hat{y}}\|_{0, \hat{K}} \text {. }
$$

Proof. (55) is satisfied if $\underline{\hat{y}}=0$. Let us suppose then that $\underline{\hat{y}} \neq 0$ and define

$$
\mathcal{F}(\underline{\hat{y}})=\min _{\underline{\hat{x}} \in X}\|\operatorname{curl} \underline{\hat{x}}+\operatorname{curl} \underline{\hat{y}}\|_{0, \hat{K}}=\left\|\operatorname{curl} \underline{\hat{y}}-P_{\operatorname{curl}(X)}(\operatorname{curl} \underline{\hat{y}})\right\|_{0, \hat{K}}
$$

where $P_{\operatorname{curl}(X)}$ is the projection operator from $\operatorname{curl}(Y)$ into $\operatorname{curl}(X)$, orthogonal with respect to $(,)_{0, \hat{K}}$. Owing to our hypothesis, curl $\underline{\hat{y}}=0$ if and only if $\underline{\hat{y}}=0$, so that we can pose $\underline{\hat{y}}^{*}=\underline{\hat{y}} / \|$ curl $\underline{\hat{y}} \|_{0, \hat{K}}$ and repeat the same logical steps used to prove Lemma 28.

Lemma 30. If $Z$ is a finite dimensional subspace of $H^{1}(\hat{K})^{3}$ such that for all $\underline{\hat{z}} \in Z$ curl $\underline{\hat{z}}=0$ implies $\underline{\hat{z}}=0$, then $\exists C>0, C$ independent of $\underline{\hat{z}}$, such that $|\underline{\hat{z}}|_{1, \hat{K}} \leq C\|\operatorname{curl} \underline{\hat{z}}\|_{0, \hat{K}}$.

Proof. We have $\operatorname{dim}(\operatorname{curl}(Z))=\operatorname{dim}(Z)<\infty$. Moreover, ||$_{1, \hat{K}}$ and \|\|$_{0, \hat{K}}$ are norms in $Z$ and $\operatorname{curl}(Z)$, respectively. Then the two spaces are topologically isomorphic and the thesis follows.

Lemma 31. Let us suppose $K \in \mathcal{T}_{h}$ is affine equivalent to $\hat{K}$. Moreover, let $\sigma>0$ be such that $\frac{h_{K}}{\rho_{K}} \leq \sigma \forall K \in \mathcal{T}_{h}$, $\forall h \in I$, and suppose that $Z \subset L^{2}(K)^{3}$ is finite dimensional and such that $B_{K}^{T}(Z)=\hat{Z}=\hat{X} \oplus \hat{Y}$. Let $X=\left(B_{K}^{T}\right)^{-1}(\hat{X})$ and $Y=\left(B_{K}^{T}\right)^{-1}(\hat{Y})$. Then $\exists C>0, C$ independent of $\underline{z}, K$, and $h$ such that

$$
\|\underline{z}\|_{0, K} \geq C\|\underline{y}\|_{0, K} \forall \underline{z} \in Z
$$

where $\underline{y}$ is the unique vector field in $Y$, such that $\underline{z}=\underline{x}+\underline{y}$, with $\underline{x} \in X$.

Proof. By using (44), Lemma 28, and (45) we have

$$
\|\underline{z}\|_{0, K} \geq C_{1}\left\|B_{K}\right\|^{-1}\left\|B_{K}^{-1}\right\|^{-1}\|\underline{y}\|_{0, K} .
$$

Then by using (48) and (49)

$$
\|\underline{z}\|_{0, K} \geq C_{1} \frac{\rho_{\hat{K}}}{h_{K}} \frac{\rho_{K}}{h_{\hat{K}}}\|\underline{y}\|_{0, K}
$$

and consequently

$$
\|\underline{z}\|_{0, K} \geq \frac{C_{1}}{\sigma} \frac{\rho_{\hat{K}}}{h_{\hat{K}}}\|\underline{y}\|_{0, K}=C\|\underline{y}\|_{0, K} .
$$


Lemma 32. Let us suppose $K \in \mathcal{T}_{h}$ is affine equivalent to $\hat{K}$. Moreover, let $\sigma>0$ be such that $\frac{h_{K}}{\rho_{K}} \leq \sigma \forall K \in \mathcal{T}_{h}$, $\forall h \in I$, and suppose that $Z \subset H(\operatorname{curl}, K)$ is finite dimensional and such that $B_{K}^{T}(Z)=\hat{Z}=\hat{X} \oplus \hat{Y}$. Let $X=\left(B_{K}^{T}\right)^{-1}(\hat{X})$ and $Y=\left(B_{K}^{T}\right)^{-1}(\hat{Y})$. If for all $\underline{\hat{z}}=\underline{\hat{x}}+\underline{\hat{y}}, \underline{\hat{x}} \in X, \underline{\hat{y}} \in Y$, curl $\underline{\hat{z}}=0$ implies $\underline{\hat{y}}=0$, then $\exists C>0, C$ independent of $\underline{z}, K$, and $h$ such that

$$
\|\operatorname{curl} \underline{z}\|_{0, K} \geq C\|\operatorname{curl} \underline{y}\|_{0, K} \forall \underline{z} \in Z
$$

where $\underline{y}$ is the unique vector field in $Y$, such that $\underline{z}=\underline{x}+\underline{y}$, with $\underline{x} \in X$.

Proof. Analogous to the previous one. Use inequality (46), Lemma 29, and inequalities (47), (48), and (49).

Lemma 33. Let us suppose $K \in \mathcal{T}_{h}$ is affine equivalent to $\hat{K}$. Moreover, let $\sigma>0$ be such that $\frac{h_{K}}{\rho_{K}} \leq \sigma \forall K \in \mathcal{T}_{h}$, $\forall h \in I$. If $Z \subset H^{1}(K)^{3}$ is finite dimensional, $\hat{Z}=B_{K}^{T}(Z)$ and for all $\underline{\hat{z}} \in \hat{Z}$, curl $\underline{\hat{z}}=0$ implies $\underline{\hat{z}}=0$, then $\exists C>0, C$ independent of $\underline{z}, K$, and $h$ such that

$$
|\underline{z}|_{1, K} \leq C\|\operatorname{curl} \underline{z}\|_{0, K} \forall \underline{z} \in Z \text {. }
$$

Proof. Analogous to the previous one. Use inequality (42), Lemma 30, and inequalities (43), (48), and (49).

Corollary 2. Under the hypotheses of Lemma 33, $\exists C>0, C$ independent of $\underline{z}$, $K$, and $h$ such that

$$
\|\underline{z}\|_{1, K} \leq C\|\underline{z}\|_{\mathrm{curl}, K} \forall \underline{z} \in Z
$$

To conclude this appendix, we now state and prove three more lemmas, which are basic to prove our main results on Nedelec's elements.

Lemma 34. Let $V \supset V_{h}=U_{h} \oplus W_{h}, \forall h \in I$. Moreover, for all $\underline{v}_{h} \in V_{h}, \underline{v}_{h}=\underline{u}_{h}+\underline{w}_{h}, \underline{u}_{h} \in U_{h}, \underline{w}_{h} \in W_{h}$, let $C_{1}, C_{2}>0, C_{1}$ and $C_{2}$ independent of $\underline{v}_{h}$ and $h$, be such that $\left\|\left.\underline{v}_{h}\right|_{K}\right\|_{0, K} \geq C_{1}\left\|\left.\underline{w}_{h}\right|_{K}\right\|_{0, K}$, and $\left\|\left.\operatorname{curl} \underline{v}_{h}\right|_{K}\right\|_{0, K} \geq$ $C_{2}\left\|\left.\operatorname{curl} \underline{w}_{h}\right|_{K}\right\|_{0, K} \forall K \in \mathcal{T}_{h}, \forall h \in I$. Then, for any sequence $\left\{\underline{v}_{h}\right\}, \underline{v}_{h} \in V_{h}, \forall h \in I$, such that $\left\|\underline{v}_{h}\right\|_{\text {curl, } \Omega} \leq C_{3}$, $\exists \exists C_{4}, C_{5}>0, C_{4}$ and $C_{5}$ independent of $\left\{\underline{v}_{h}\right\}$, such that $\left\|\underline{u}_{h}\right\|_{\mathrm{curl}, \Omega} \leq C_{4}$ and $\left\|\underline{w}_{h}\right\|_{\mathrm{curl}, \Omega} \leq C_{5}$.

Proof. As $\|a+b\| \geq|\|a\|-\|b\||$ the thesis can be wrong only if both sequences, $\left\{\left\|\underline{u}_{h}\right\|_{\text {curl }, \Omega}\right\}$ and $\left\{\left\|\underline{w}_{h}\right\|_{\text {curl }, \Omega}\right\}$, are unbounded. But

$$
\begin{aligned}
\left\|\underline{v}_{h}\right\|_{\mathrm{curl}, \Omega}^{2} & =\sum_{K \in \mathcal{T}_{h}}\left\|\left.\underline{v}_{h}\right|_{K}\right\|_{\mathrm{curl}, K}^{2} \\
& =\sum_{K \in \mathcal{T}_{h}}\left\|\left.\underline{v}_{h}\right|_{K}\right\|_{0, K}^{2}+\sum_{K \in \mathcal{T}_{h}}\left\|\operatorname{curl}\left(\left.\underline{v}_{h}\right|_{K}\right)\right\|_{0, K}^{2} \\
& \geq \sum_{K \in \mathcal{T}_{h}} C_{1}^{2}\left\|\left.\underline{w}_{h}\right|_{K}\right\|_{0, K}^{2}+\sum_{K \in \mathcal{T}_{h}} C_{2}^{2}\left\|\left.\operatorname{curl} \underline{w}_{h}\right|_{K}\right\|_{0, K}^{2} \\
& =\max \left(C_{1}^{2}, C_{2}^{2}\right)\left\|_{w_{h}}\right\|_{\text {curl }, \Omega}^{2}
\end{aligned}
$$

and, the boundedness of $\left\|\underline{w}_{h}\right\|_{\text {curl }, \Omega}$ implies that of $\left\|\underline{w}_{h}\right\|_{\text {curl }, \Omega}$.

Lemma 35. Assume that $U_{h}=\left\{\underline{u}_{h} \in V:\left.\underline{u}_{h}\right|_{K} \in U \forall K \in \mathcal{T}_{h}\right\}$, where $U \subset H(\operatorname{curl}, K)$ is invariant under transformation (27) and such that $\operatorname{grad}\left(P_{1}\right) \subset U$. Then $\operatorname{grad}\left(P_{1, h}\right) \subset U_{h}$. If, moreover, $\exists s>1 / 2$ such that $\mathbb{H} \subset H^{s}(\Omega)^{3}$, then $\left\{U_{h}\right\}$ satisfies $(C D K)$.

Proof. By (14) and (13), $\forall p_{h} \in P_{1, h} \quad \operatorname{grad} p_{h} \in V$. Moreover, $\left.\forall K \in \mathcal{T}_{h} \operatorname{grad} p_{h}\right|_{K} \in \operatorname{grad}\left(P_{1}\right) \subset U$. Hence, $\operatorname{grad} p_{h} \in U_{h}$ and the first part is proved. 
Let us define $U_{0 h}=U_{h} \cap V_{0}$. Owing to (14), in order to prove that $\left\{U_{h}\right\}$ satisfies (CDK), we can separately prove

$$
\begin{gathered}
\forall \underline{u} \in \operatorname{grad}\left(H_{0, \Gamma_{\tau}}^{1}(\Omega)\right) \lim _{h \rightarrow 0} \inf _{h} \in U_{0 h}\left\|\underline{u}-\underline{u}_{h}\right\|_{0, \Omega}=0 \\
\forall \underline{v} \in \mathbb{H} \lim _{h \rightarrow 0} \underline{\inf }_{h} \in U_{0 h} \\
\left\|\underline{v}-\underline{u}_{h}\right\|_{0, \Omega}=0 .
\end{gathered}
$$

We have

$$
\inf _{\underline{u}_{h} \in \operatorname{grad}\left(P_{1, h}\right)}\left\|\underline{u}-\underline{u}_{h}\right\|_{0, \Omega}=\inf _{p_{h} \in P_{1, h}}\left\|\operatorname{grad} p-\operatorname{grad} p_{h}\right\|_{0, \Omega} \leq \inf _{p_{h} \in P_{1, h}}\left\|p-p_{h}\right\|_{1, \Omega} .
$$

Since, by a standard result for scalar Lagrangian elements, the last term tends to zero as $h \rightarrow 0$ and, moreover, $\operatorname{grad}\left(P_{1, h}\right) \subset U_{h}$ and $\operatorname{grad}\left(P_{1, h}\right) \subset V_{0},(65)$ immediately follows.

As any $\underline{v} \in \mathbb{H}$ admits a scalar potential taking constant values on the connected components of $\Gamma_{\tau}$ and having constant jumps across the cuts [19], with our assumptions about cuts and triangulations we have that $\forall h \in I \exists \phi^{(h)}$ such that $\underline{v}=\operatorname{grad} \phi^{(h)}$ in $\dot{\Omega}_{h}$. As $\underline{v} \in H^{s}(\Omega)^{3}$ we have $\left.\underline{v}\right|_{\dot{\Omega}_{h}} \in H^{s}\left(\dot{\Omega}_{h}\right)^{3}$ and, consequently, $\phi^{(h)} \in H^{1+s}\left(\dot{\Omega}_{h}\right)$ with $s>1 / 2$.

Let us define (see [16] and [25]) $\pi_{K}: H^{1+s}(K) \rightarrow P_{1}$ by $\left(\pi_{K} \psi\right)\left(\underline{x}_{j}\right)=\psi\left(\underline{x}_{j}\right), \underline{x}_{j}$ being the vertices of the element $K \in \mathcal{T}_{h}$, and then $\pi_{h}: H^{1+s}\left(\dot{\Omega}_{h}\right) \rightarrow\left\{p_{h} \in H^{1}\left(\dot{\Omega}_{h}\right):\left.p_{h}\right|_{K} \in P_{1} \forall K \in \mathcal{T}_{h}\right\}$ by $\left.\left(\pi_{h} \psi\right)\right|_{K}=\pi_{K}\left(\left.\psi\right|_{K}\right) \forall K \in$ $\mathcal{T}_{h}$

Let us introduce $\underline{v}_{h}=\operatorname{grad} \pi_{h} \phi^{(h)} \in L^{2}\left(\dot{\Omega}_{h}\right)^{3}$, which can be extended to $L^{2}(\Omega)^{3}$. As $\pi_{h}$ preserves both constant values on the connected components of $\Gamma_{\tau}$ and constant jumps across $\Sigma_{j, h}, \underline{v}_{h} \in V$.

Moreover, $\left.\underline{v}_{h}\right|_{K}=\left.\operatorname{grad} \pi_{h} \phi^{(h)}\right|_{K} \in \operatorname{grad}\left(P_{1}\right) \subset U$. Hence, $\underline{v}_{h} \in U_{h}$.

Finally, as curl $\underline{v}_{h}=0, \underline{v}_{h} \in U_{0 h}$.

Now, we have

$$
\inf _{\underline{u}_{h} \in U_{0 h}}\left\|\underline{v}-\underline{u}_{h}\right\|_{0, \Omega} \leq\left\|\underline{v}-\underline{v}_{h}\right\|_{0, \Omega}=\left\|\operatorname{grad} \phi^{(h)}-\operatorname{grad} \pi_{h} \phi^{(h)}\right\|_{0, \Omega}=\left\|\operatorname{grad} \phi^{(h)}-\operatorname{grad} \pi_{h} \phi^{(h)}\right\|_{0, \dot{\Omega}_{h}} .
$$

As $\Sigma_{h}$ does not cut any element of the triangulation, Lemma 3 of [25] holds true $\forall K \in \mathcal{T}_{h}$. Thus, the estimate of Lemma 4 of [25] applies as well and gives

$$
\left\|\operatorname{grad} \phi^{(h)}-\operatorname{grad} \pi_{h} \phi^{(h)}\right\|_{0, \dot{\Omega}_{h}} \leq C h^{s}\left(\sum_{K \in \mathcal{T}_{h}}\left\|\left.\operatorname{grad} \phi^{(h)}\right|_{K}\right\|_{s, K}^{2}\right)^{1 / 2}=C h^{s}\left(\sum_{K \in \mathcal{T}_{h}}\left\|\left.\underline{v}\right|_{K}\right\|_{s, K}^{2}\right)^{1 / 2}=C h^{s}\|\underline{v}\|_{s, \Omega}
$$

Finally, (66) immediately follows from

$$
\inf _{\underline{u}_{h} \in U_{0 h}}\left\|\underline{v}-\underline{u}_{h}\right\|_{0, \Omega} \leq C h^{s}\|\underline{v}\|_{s, \Omega} .
$$

Lemma 36. Let $V \supset V_{h}=U_{h} \oplus W_{h}, \forall h \in I$, and suppose that $\forall \underline{w}_{h} \in W_{h}$ we have $\left.\underline{w}_{h}\right|_{K} \in H^{1}(K)^{3} \forall K \in$ $\mathcal{T}_{h}, \forall h \in I$, and that $\left\|\left.\underline{w}_{h}\right|_{K}\right\|_{1, K} \leq C_{1}\left\|\left.\underline{w}_{h}\right|_{K}\right\|_{\text {curl }, K}$. Moreover, for all $\left\{\underline{v}_{h}\right\}, \underline{v}_{h} \in V_{h} \forall h \in I, \underline{v}_{h}=\underline{u}_{h}+\underline{w}_{h}$, where $\underline{u}_{h} \in U_{h}$ and $\underline{w}_{h} \in W_{h}$, suppose that $\left\|\underline{v}_{h}\right\|_{\text {curl }, \Omega} \leq C$ implies either $\left\|\underline{u}_{h}\right\|_{\text {curl }, \Omega} \leq C_{2}$ or $\left\|\underline{w}_{h}\right\|_{\text {curl }, \Omega} \leq$ $C_{3}$. Finally, suppose that $\exists s>1 / 2$ such that $V_{1}$ and $\mathbb{H} \subset H^{s}(\Omega)^{3}$ and that $\exists C_{4}>0$ such that $\forall \underline{v}_{1} \in V_{1}$, $\left\|\underline{v}_{1}\right\|_{s, \Omega} \leq C_{4}\left\|\underline{v}_{1}\right\|_{\text {curl }, \Omega}$. Then $\left\{V_{h}\right\}$ satisfies (DCP) (with strong limit in $V_{1}$ ) if $\left\{U_{h}\right\}$ satisfies $(C D K)$, (DCP) and $\operatorname{grad}\left(P_{1, h}\right) \subset U_{h} \forall h \in I$. 
Proof. Let us consider a sequence $\left\{\underline{v}_{h}\right\}, \underline{v}_{h} \in V_{1 h},\left\|\underline{v}_{h}\right\|_{\text {curl, } \Omega} \leq C$. We know that $\underline{v}_{h}=\underline{u}_{h}+\underline{w}_{h}$. But, if $U_{0 h}=U_{h} \cap V_{0}$ and $U_{1 h}$ is its orthogonal (with respect to the (, ) curl, $\Omega$ scalar product) complement in $U_{h}$, we have $U_{h}=U_{0 h} \oplus U_{1 h}$. Then $\underline{v}_{h}=\underline{u}_{1 h}+\underline{u}_{0 h}+\underline{w}_{h}, \underline{u}_{1 h} \in U_{1 h}, \underline{u}_{0 h} \in U_{0 h}$. Thanks to our hypotheses both $\underline{u}_{h}$ and $\underline{w}_{h}$ are bounded and then $\underline{u}_{1 h}$ is bounded, too. As $\left\{U_{h}\right\}$ satisfies (DCP) we have that there exists a subsequence of $\left\{\underline{u}_{1 h}\right\}$ (still denoted by $\left\{\underline{u}_{1 h}\right\}$ ) such that $\underline{u}_{1 h} \rightarrow \underline{u}_{1}$ strongly in $H$. But $\left\{U_{h}\right\}$ satisfies also (CDK) and, consequently, by Proposition 2.18 of [13], $\underline{u}_{1} \in V_{1}$. Moreover it is always possible to write $\underline{w}_{h}=\underline{w}_{0, c, h}+\underline{w}_{1, c, h}, \underline{w}_{0, c, h} \in V_{0}$ and $\underline{w}_{1, c, h} \in V_{1}$. The boundedness of $\underline{w}_{h}$ and the compact embedding of $V_{1}$ into $H$ imply that there exists a subsequence of $\left\{\underline{w}_{1, c, h}\right\}$ (still denoted by $\left\{\underline{w}_{1, c, h}\right\}$ ) such that $\underline{w}_{1, c, h} \rightarrow \underline{w}_{1}$ strongly in $H, \underline{w}_{1} \in V_{1}$. As $\left.\underline{w}_{h}\right|_{K} \in H^{1}(K)^{3} \forall K \in \mathcal{T}_{h}$ and $\exists s>1 / 2$ such that $V_{1} \subset H^{s}(\Omega)^{3}$ we have $\left.\underline{w}_{0, c, h}\right|_{K} \in H^{s}(K)^{3}$. But $\underline{w}_{0, c, h}=\underline{w}_{\mathbb{H}, c, h}+\underline{w}_{g, c, h}$ where $\underline{w}_{\mathbb{H}, c, h}$ belongs to $\mathbb{H} \subset H^{s}(\Omega)^{3}$. Therefore $\left.\underline{w}_{g, c, h}\right|_{K} \in H^{s}(K)^{3}$, and, as $\underline{w}_{g, c, h}=\operatorname{grad} p, p \in H_{0, \Gamma_{\tau}}^{1}(\Omega)[19]$, we obtain $\left.p\right|_{K} \in H^{1+s}(K)$. Note that $\mathbb{H}$ is closed in $V$ [19] and has a finite dimension [19]. As the sequence $\left\{\underline{w}_{\mathbb{H}, c, h}\right\}$ is bounded, there exists a subsequence (still denoted by $\left\{\underline{w}_{\mathbb{H}, c, h}\right\}$ ) such that $\exists \underline{v}_{\mathbb{H}} \in \mathbb{H}: \underline{w}_{\mathbb{H}, c, h} \rightarrow \underline{v}_{\mathbb{H}}$ in $V$. As $\left\{U_{h}\right\}$ satisfies (CDK) $\exists\left\{\underline{u}_{0 h}^{*}\right\}, \underline{u}_{0 h}^{*} \in U_{0 h}, \forall h \in I$, such that $\underline{u}_{0 h}^{*} \rightarrow \underline{v}_{\mathbb{H}}$ in $V$. Moreover, $\operatorname{grad}\left(P_{1, h}\right) \subset U_{0 h} \forall h \in I$ and consequently, by Lemma 4 of $[25] \exists\left\{\underline{u}_{0 h, *}\right\}, \underline{u}_{0 h, *} \in \operatorname{grad}\left(P_{1, h}\right) \subset U_{0 h}$ and $\exists C_{5}>0, C_{5}$ independent of $\underline{w}_{g, c, h}$ and $h$ such that

$$
\left\|\underline{w}_{g, c, h}-\underline{u}_{0 h, *}\right\|_{0, \Omega} \leq C_{5} h^{s}\left(\sum_{K \in \mathcal{T}_{h}}\left\|\left.\underline{w}_{g, c, h}\right|_{K}\right\|_{s, K}^{2}\right)^{1 / 2} .
$$

But

$$
\begin{aligned}
\left\|\left.\underline{w}_{g, c, h}\right|_{K}\right\|_{s, K}^{2} & \leq 2\left\|\left.\underline{w}_{0, c, h}\right|_{K}\right\|_{s, K}^{2}+2\left\|\underline{w}_{\mathbb{H}, c, h}||_{K}\right\|_{s, K}^{2}=2\left\|\left.\underline{w}_{h}\right|_{K}-\left.\underline{w}_{1, c, h}\right|_{K}\right\|_{s, K}^{2}+2\left\|\left.\underline{w}_{\mathbb{H}, c, h}\right|_{K}\right\|_{s, K}^{2} \\
& \leq 4\left\|\left.\underline{w}_{h}\right|_{K}\right\|_{s, K}^{2}+4\left\|\left.\underline{w}_{1, c, h}\right|_{K}\right\|_{s, K}^{2}+2\left\|\left.\underline{w}_{\mathbb{H}, c, h}\right|_{K}\right\|_{s, K}^{2} .
\end{aligned}
$$

Consequently

$$
\begin{aligned}
\sum_{K \in \mathcal{T}_{h}}\left\|\underline{w}_{g, c, h}||_{K}\right\|_{s, K}^{2} & \leq 4 \sum_{K \in \mathcal{T}_{h}}\left\|\left.\underline{w}_{h}\right|_{K}\right\|_{s, K}^{2}+4 \sum_{K \in \mathcal{T}_{h}}\left\|\left.\underline{w}_{1, c, h}\right|_{K}\right\|_{s, K}^{2}+2 \sum_{K \in \mathcal{T}_{h}}\left\|\left.\underline{w}_{\mathbb{H}, c, h}\right|_{K}\right\|_{s, K}^{2} \\
& \leq 4 \sum_{K \in \mathcal{T}_{h}}\left\|\left.\underline{w}_{h}\right|_{K}\right\|_{1, K}^{2}+4\left\|\underline{w}_{1, c, h}\right\|_{s, \Omega}^{2}+2\left\|\underline{w}_{\mathbb{H}, c, h}\right\|_{s, \Omega}^{2} .
\end{aligned}
$$

As already pointed out $\left\{\underline{w}_{\mathbb{H}, c, h}\right\}$ is bounded in $V, \underline{w}_{\mathbb{H}, c, h} \in \mathbb{H}, \forall h \in I$, and $\mathbb{H}$ is finite dimensional. Moreover, $\mathbb{H} \subset H^{s}(\Omega)^{3}$. Then \|\|$_{s, \Omega}$ and \|\|$_{V, \Omega}$ are equivalent in $\mathbb{H}$. Therefore $\exists C_{6}$ such that $\left\|\underline{w}_{\mathbb{H}, c, h}\right\|_{s, \Omega} \leq C_{6}$ and

$$
\sum_{K \in \mathcal{T}_{h}}\left\|\left.\underline{w}_{g, c, h}\right|_{K}\right\|_{s, K}^{2} \leq 4 \sum_{K \in \mathcal{T}_{h}}\left\|\left.\underline{w}_{h}\right|_{K}\right\|_{1, K}^{2}+4\left\|\underline{w}_{1, c, h}\right\|_{s, \Omega}^{2}+2 C_{6}^{2}
$$

Finally, we can now conclude that

$$
\begin{aligned}
\sum_{K \in \mathcal{T}_{h}}\left\|\left.\underline{w}_{g, c, h}\right|_{K}\right\|_{s, K}^{2} & \leq 4 \sum_{K \in \mathcal{T}_{h}} C_{1}^{2}\left\|\left.\underline{w}_{h}\right|_{K}\right\|_{\mathrm{curl}, K}^{2}+4 C_{4}^{2}\left\|\underline{w}_{1, c, h}\right\|_{\mathrm{curl}, \Omega}^{2}+2 C_{6}^{2} \\
& \leq 4 C_{1}^{2}\left\|\underline{w}_{h}\right\|_{\mathrm{curl}, \Omega}^{2}+4 C_{4}^{2}\left\|\underline{w}_{h}\right\|_{\mathrm{curl}, \Omega}^{2}+2 C_{6}^{2} \leq C_{7}^{2} .
\end{aligned}
$$

Then

$$
\left\|\underline{w}_{g, c, h}-\underline{u}_{0 h, *}\right\|_{0, \Omega} \leq C_{5} C_{7} h^{s} .
$$


Let us consider now $\underline{v}_{0 h}=\underline{u}_{0 h}+\underline{u}_{0 h, *}+\underline{u}_{0 h}^{*}, \underline{v}_{0 h} \in U_{0 h}$. We have

$$
\left(\underline{v}_{h}, \underline{v}_{0 h}\right)_{0, \Omega}=\left(\underline{u}_{1 h}, \underline{v}_{0 h}\right)_{0, \Omega}+\left(\underline{u}_{0 h}, \underline{v}_{0 h}\right)_{0, \Omega}+\left(\underline{w}_{1, c, h}, \underline{v}_{0 h}\right)_{0, \Omega}+\left(\underline{w}_{0, c, h}, \underline{v}_{0 h}\right)_{0, \Omega} .
$$

But $\left(\underline{v}_{h}, \underline{v}_{0 h}\right)_{0, \Omega}=0$ as $\underline{v}_{0 h} \in U_{0 h} \subset V_{0 h},\left(\underline{u}_{1 h}, \underline{v}_{0 h}\right)_{0, \Omega}=0$ as $\underline{v}_{0 h} \in U_{0 h}$ and $\underline{u}_{1 h} \in U_{1 h}$ and $\left(\underline{w}_{1, c, h}, \underline{v}_{0 h}\right)_{0, \Omega}=0$ as $\underline{w}_{1, c, h} \in V_{1}$ and $\underline{v}_{0 h} \in V_{0}$. Then

$$
\begin{aligned}
0 & =\left(\underline{u}_{0 h}, \underline{v}_{0 h}\right)_{0, \Omega}+\left(\underline{w}_{0, c, h}, \underline{v}_{0 h}\right)_{0, \Omega}=\left(\underline{u}_{0 h}+\underline{w}_{0, c, h}, \underline{v}_{0 h}\right)_{0, \Omega} \\
& =\left(\underline{u}_{0 h}+\underline{w}_{0, c, h}-\underline{u}_{0 h, *}+\underline{u}_{0 h, *}-\underline{u}_{0 h}^{*}+\underline{u}_{0 h}^{*}, \underline{v}_{0 h}\right)_{0, \Omega} \\
& =\left(\underline{v}_{0 h}, \underline{v}_{0 h}\right)_{0, \Omega}+\left(\underline{w}_{0, c, h}-\underline{u}_{0 h, *}-\underline{u}_{0 h}^{*}, \underline{v}_{0 h}\right)_{0, \Omega}
\end{aligned}
$$

which implies

$$
\begin{aligned}
\left\|\underline{v}_{0 h}\right\|_{0, \Omega}^{2} & =-\left(\underline{w}_{0, c, h}-\underline{u}_{0 h, *}-\underline{u}_{0 h}^{*}, \underline{v}_{0 h}\right)_{0, \Omega} \\
& =\left|\left(\underline{w}_{0, c, h}-\underline{u}_{0 h, *}-\underline{u}_{0 h}^{*}, \underline{v}_{0 h}\right)_{0, \Omega}\right| \leq\left\|\underline{w}_{0, c, h}-\underline{u}_{0 h, *}-\underline{u}_{0 h}^{*}\right\|_{0, \Omega}\left\|\underline{v}_{0 h}\right\|_{0, \Omega}
\end{aligned}
$$

i.e.,

$$
\begin{array}{r}
\left\|\underline{v}_{0 h}\right\|_{0, \Omega} \leq\left\|\underline{w}_{0, c, h}-\underline{u}_{0 h, *}-\underline{u}_{0 h}^{*}\right\|_{0, \Omega}=\left\|\underline{w}_{\mathbb{H}, c, h}-\underline{u}_{0 h}^{*}+\underline{w}_{g, c, h}-\underline{u}_{0 h, *}\right\|_{0, \Omega} \\
\leq\left\|\underline{w}_{\mathbb{H}, c, h}-\underline{u}_{0 h}^{*}\right\|_{0, \Omega}+\left\|\underline{w}_{g, c, h}-\underline{u}_{0 h, *}\right\|_{0, \Omega}
\end{array}
$$

Then also $\left\|\underline{v}_{0 h}\right\|_{0, \Omega} \rightarrow 0$. It then follows that $\underline{w}_{0, c, h}+\underline{u}_{0 h}=\underline{v}_{0 h}+\underline{w}_{0, c, h}-\underline{u}_{0 h, *}-\underline{u}_{0 h}^{*} \rightarrow 0$, as both $\left\|\underline{v}_{0 h}\right\|_{0, \Omega} \rightarrow 0$ and $\left\|\underline{w}_{0, c, h}-\underline{u}_{0 h, *}-\underline{u}_{0 h}^{*}\right\|_{0, \Omega} \rightarrow 0$. Consequently, there exists a subsequence of $\left\{\underline{v}_{h}\right\}$ (still denoted by $\left\{\underline{v}_{h}\right\}$ ) such that $\underline{v}_{h}=\underline{u}_{1 h}+\underline{u}_{0 h}+\underline{w}_{1, c, h}+\underline{w}_{0, c, h} \rightarrow \underline{u}_{1}+\underline{w}_{1}$ strongly in $H, \underline{u}_{1}+\underline{w}_{1} \in V_{1}$.

Remark 7. Owing to Lemma 35, in the statement of Lemma 36 we may substitute the hypotheses $\left\{U_{h}\right\}$ satisfies $(\mathrm{CDK})$ and $\operatorname{grad}\left(P_{1, h}\right) \subset U_{h} \forall h \in I$ by the hypotheses $U_{h}=\left\{\underline{u}_{h} \in V:\left.\underline{u}_{h}\right|_{K} \in U \forall K \in \mathcal{T}_{h}\right\}$, where $U \subset H(\operatorname{curl}, K)$ is invariant under transformation (27) and such that $\operatorname{grad}\left(P_{1}\right) \subset \bar{U}$.

\section{REFERENCES}

[1] C. Amrouche, C. Bernardi, M. Dauge and V. Girault, Vector potential in three-dimensional nonsmooth domains. Math. Methods Appl. Sci. 21 (1998) 823-864.

[2] M.L. Barton and Z.J. Cendes, New vector finite elements for three-dimensional magnetic field computation. J. Appl. Phys. 61 (1987) 3919-3921.

[3] A. Bermudez and D.G. Pedreira, Mathematical analysis of a finite element method without spurious solutions for computation of dielectric waveguides. Numer. Math. 61 (1992) 39-57.

[4] D. Boffi, Fortin operator and discrete compactness for edge elements. Numer. Math. 86 (2000). DOI 10.1007/s002110000182.

[5] D. Boffi, A note on the discrete compactness property and the de Rham complex. Technical Report AM188, Department of Mathematics, Penn State University, 1999. Appl. Math. Lett. 14 (2001) 33-38.

[6] D. Boffi, P. Fernandes, L. Gastaldi and I. Perugia, Computational models of electromagnetic resonators: analysis of edge element approximation. SIAM J. Numer. Anal. 36 (1999) 1264-1290.

[7] A. Bossavit, Whitney forms: a class of finite elements for three-dimensional computations in electromagnetism. IEE Proceedings, part A 135 (1988) 493-500.

[8] A. Bossavit, A rationale for 'edge-elements' in 3-D fields computations. IEEE Trans. Magnet. 24 (1988) 74-79.

[9] A. Bossavit, Solving maxwell's equations in a closed cavity, and the question of 'spurious modes'. IEEE Trans. Magnet. 26 (1990) 702-705.

[10] S. Caorsi, P. Fernandes and M. Raffetto, Edge elements and the inclusion condition. IEEE Microwave Guided Wave Lett. 5 (1995) 222-223.

[11] S. Caorsi, P. Fernandes and M. Raffetto, Towards a good characterization of spectrally correct finite element methods in electromagnetics. COMPEL 15 (1996) 21-35. 
[12] S. Caorsi, P. Fernandes and M. Raffetto, Do covariant projection elements really satisfy the inclusion condition? IEEE Trans. Microwave Theory Tech. 45 (1997) 1643-1644.

[13] S. Caorsi, P. Fernandes and M. Raffetto, On the convergence of Galerkin finite element approximations of electromagnetic eigenproblems. SIAM J. Numer. Anal. 38 (2000) 580-607.

[14] S. Caorsi, P. Fernandes and M. Raffetto, Characteristic conditions for spurious-free finite element approximations of electromagnetic eigenproblems, in Proceedings of ECCOMAS 2000, Barcelona, Spain (2000) 1-13.

[15] Z.J. Cendes and P.P. Silvester, Numerical solution of dielectric loaded waveguides: I-finite-element analysis. IEEE Trans. Microwave Theory Tech. 18 (1970) 1124-1131.

[16] P.G. Ciarlet, The finite element method for elliptic problems. North-Holland, Amsterdam (1978).

[17] C.W. Crowley, P.P. Silvester and H. Hurwitz Jr., Covariant projection elements for 3d vector field problems. IEEE Trans. Magnet. 24 (1988) 397-400.

[18] J.B. Davies, F.A. Fernandez and G.Y. Philippou, Finite element analysis of all modes in cavities with circular symmetry. IEEE Trans. Microwave Theory Tech. 30 (1982) 1975-1980.

[19] P. Fernandes and G. Gilardi, Magnetostatic and electrostatic problems in inhomogeneous anisotropic media with irregular boundary and mixed boundary conditions. Math. Models Methods Appl. Sci. 7 (1997) 957-991.

[20] V. Girault and P.A. Raviart, Finite element methods for Navier-Stokes equations. Springer-Verlag, Berlin (1986).

[21] M. Hano, Vector finite element solution of anisotropic waveguides using novel triangular elements. Electron. Com. Japan, Part 2, 71 (1988) 71-80.

[22] M. Hara, T. Wada, T. Fukasawa and F. Kikuchi, A three dimensional analysis of rf electromagnetic fields by the finite element method. IEEE Trans. Magnet. 19 (1983) 2417-2420.

[23] H.C. Hoyt, Numerical studies of the shapes of drift tubes and Linac cavities. IEEE Trans. Nucl. Sci. 12 (1965) $153-155$.

[24] H.C. Hoyt, D.D. Simmonds and W.F. Rich, Computer designed $805 \mathrm{MHz}$ proton Linac cavities. The Review of Scientific Instruments 37 (1966) 755-762.

[25] F. Kikuchi, On a discrete compactness property for the Nedelec finite elements. J. Fac. Sci., Univ. Tokyo 36 (1989) $479-490$.

[26] F. Kikuchi, Theoretical analysis of Nedelec's edge elements, in Proceedings of Computational Engineering Conference, Tokyo, Japan, May 26-28 (1999).

[27] R. Miniowitz and J.P. Webb, Covariant-projection quadrilateral elements for the analysis of waveguides with sharp edges. IEEE Trans. Microwave Theory Tech. 39 (1991) 501-505.

[28] P. Monk and L. Demkowicz, Discrete compactness and the approximation of Maxwell's equations in $\mathbb{R}^{3}$. Math. Comput. 70 (2001) 507-523.

[29] J.C. Nedelec, Mixed finite elements in $\mathbb{R}^{3}$. Numer. Math. 35 (1980) 315-341.

[30] J.C. Nedelec, A new family of mixed finite elements in $\mathbb{R}^{3}$. Numer. Math. 50 (1986) 57-81.

[31] R. Parodi, A. Stella and P. Fernandes, Rf tests of a band overlap free daw accelerating structure, in Proceedings of the IEEE 1991 Particle Accelerator Conference, San Francisco, USA (1991) 3026-3028.

[32] J.S. Wang and N. Ida, Curvilinear and higher order 'edge' finite elements in electromagnetic field computation. IEEE Trans. Magnet. 29 (1993) 1491-1494.

[33] S.H. Wong and Z.J. Cendes, Combined finite element-modal solution of three-dimensional eddy current problems. IEEE Trans. Magnet. 24 (1988) 2685-2687.

[34] J.P. Webb, Hierarchal vector basis functions of arbitrary order for triangular and tetrahedral finite elements. IEEE Trans. Antennas Propagation 47 (1999) 1244-1253

[35] J.P. Webb and R. Miniowitz, Analysis of 3-D microwave resonators using covariant-projection elements. IEEE Trans. Microwave Theory Tech. 39 (1991) 1895-1899.

To access this journal online:

www.edpsciences.org 